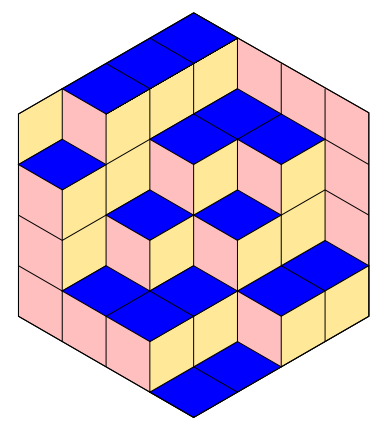

James East, Maximilien Gadouleau \& James D. Mitchell

Structural aspects of semigroups based on digraphs

Volume 2, issue 5 (2019), p. 711-733.

<http://alco.centre-mersenne.org/item/ALCO_2019__2_5_711_0>

(C) The journal and the authors, 2019.

Some rights reserved.

(c) BY This article is licensed under the

Creative Commons ATtribution 4.0 InTERnational License.

http://creativecommons.org/licenses/by/4.0/

Access to articles published by the journal Algebraic Combinatorics on the website http://alco.centre-mersenne.org/ implies agreement with the Terms of Use (http://alco.centre-mersenne.org/legal/).

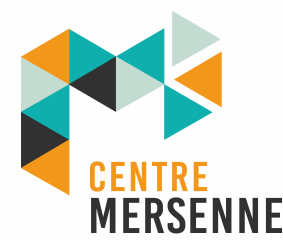

Algebraic Combinatorics is member of the Centre Mersenne for Open Scientific Publishing www.centre-mersenne.org 


\title{
Structural aspects of semigroups based on digraphs
}

\author{
James East, Maximilien Gadouleau \& James D. Mitchell
}

\begin{abstract}
Given any digraph $D$ without loops or multiple arcs, there is a natural construction of a semigroup $\langle D\rangle$ of transformations. To every arc $(a, b)$ of $D$ is associated the idempotent transformation $(a \rightarrow b)$ mapping $a$ to $b$ and fixing all vertices other than $a$. The semigroup $\langle D\rangle$ is generated by the idempotent transformations $(a \rightarrow b)$ for all $\operatorname{arcs}(a, b)$ of $D$.

In this paper, we consider the question of when there is a transformation in $\langle D\rangle$ containing a large cycle, and, for fixed $k \in \mathbb{N}$, we give a linear time algorithm to verify if $\langle D\rangle$ contains a transformation with a cycle of length $k$. We also classify those digraphs $D$ such that $\langle D\rangle$ has one of the following properties: inverse, completely regular, commutative, simple, 0 -simple, a semilattice, a rectangular band, congruence-free, is $\mathcal{K}$-trivial or $\mathcal{K}$-universal where $\mathcal{K}$ is any of Green's $\mathcal{H}-, \mathcal{L}-, \mathcal{R}$-, or $\mathcal{J}$-relation, and when $\langle D\rangle$ has a left, right, or two-sided zero.
\end{abstract}

\section{INTRODUCTION}

A transformation of degree $n \in \mathbb{N}$ is a function from $\{1, \ldots, n\}$ to itself. A transformation semigroup is a semigroup consisting of transformations of equal degree and with the operation of composition of functions. For the sake of brevity we will denote $\{1, \ldots, n\}$ by $[n]$. We define $(a \rightarrow b)$ to be the transformation defined by

$$
v(a \rightarrow b)= \begin{cases}b & \text { if } v=a \\ v & \text { otherwise }\end{cases}
$$

where $a, b \in[n]$ and $a \neq b$. A digraph is an ordered pair $(V, A)$, where $V$ is a set whose elements are referred to as vertices, and $A \subseteq(V \times V) \backslash\{(v, v): v \in V\}$ is a set of ordered pairs called arcs. We identify a transformation $(a \rightarrow b)$ with an arc $(a, b)$ in a digraph and we refer to $(a \rightarrow b)$ as an arc. If $D$ is a digraph, we denote by $\langle D\rangle$ the semigroup generated by the $\operatorname{arcs}$ of $D$, and we refer to such a semigroup as arc-generated.

If $A$ is empty, then $D$ is an empty digraph, and $\langle D\rangle$ is the empty semigroup. Because this is a degenerate case, and in order to simplify the statement of our results, we shall always assume that $D$ is not empty (and hence, that $\langle D\rangle$ is not the empty semigroup).

Arc-generated semigroups were first introduced by John Rhodes in the 1960s [20, Definition 6.51], under the name semigroups of flows. In [20], Rhodes was largely concerned with determining the maximal subgroups of an arc-generated semigroup, and he conjectured that every such subgroup was isomorphic to a direct product of

Manuscript received 2nd March 2018, revised 26th November 2018 and 21st December 2018, accepted 21st December 2018.

KEYwORDS. digraphs, flow semigroup of digraph, semigroups, monoids. 
cyclic, alternating, and symmetric groups. This conjecture was recently proved in a remarkable paper [11] by Horváth, Nehaniv, and Podoski.

Arc-generated semigroups are also closely related to the pebble motion problem on graphs [16], which we briefly describe here. Let $G=(V, E)$ be a graph on $n$ vertices and $P=\{1, \ldots, k\}$ be a set of pebbles, with $k<n$. An arrangement of pebbles is an injective mapping $S: P \rightarrow V$, which places every pebble on a different vertex. A move then consists of transferring a pebble $p \in P$ from a vertex $u$ to an adjacent vertex $v$, provided there is no pebble present in $v$ already. The main problem is, given two arrangements of pebbles $S$ and $S^{\prime}$, to determine whether one can go from $S$ to $S^{\prime}$ by using a sequence of moves. A canonical example of the pebble motion problem is the fifteen-puzzle on graphs [23], which corresponds to the case $k=n-1$. Clearly, moving a pebble from $u$ to $v$ corresponds to applying the arc $(u \rightarrow v)$; as such, we shall use some of the results in [23] later in this paper. Conversely, one of our main results, namely Theorem 3.15, can be applied to the pebble motion problem.

Many famous examples of semigroups are arc-generated. Perhaps the best known example is the semigroup $\operatorname{Sing}_{n}$ of all non-invertible, or singular, transformations on $[n]$, which was shown to be arc-generated by J. M. Howie in [12]. Other examples include the semigroup of singular order-preserving transformations [1], and the socalled Catalan semigroup $[9,21]$, which are generated by the arcs of the digraphs $\{(i, i+1),(i+1, i): i \in\{1, \ldots, n-1\}\}$ and $\{(i, i+1): i \in\{1, \ldots, n-1\}\}$, respectively; these digraphs can be seen in Figure 1 .

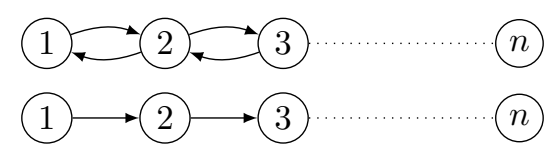

Figure 1. The digraphs $D$ where $\langle D\rangle$ is the semigroup of singular order-preserving transformations (top) or the Catalan semigroup (bottom).

In [13], Howie showed that $\operatorname{Sing}_{n}$ is generated by $\frac{1}{2} n(n-1)$, but no fewer, arcs. In [8] it was shown that $\frac{1}{2} n(n-1)$ is the minimum size of any generating set for $\operatorname{Sing}_{n}$ whether it consists of arcs or not. It was shown in [13] that $\operatorname{Sing}_{n}$ is generated by the $\operatorname{arcs}$ of a digraph $D$ if and only if $D$ is strongly connected and $D$ contains a tournament. As a corollary, the minimal-size idempotent generating sets of $\operatorname{Sing}_{n}$ are in one-one correspondence with the strongly connected tournaments on $n$ vertices; these were enumerated by Wright [24]. In [6] it was shown that a digraph $D$ is strongly connected and contains a tournament if and only if it contains a strongly connected tournament. Hence every idempotent generating set for $\operatorname{Sing}_{n}$ contains one of minimum size, something that is not true for generating sets of Sing ${ }_{n}$, in general.

Several authors have classified those digraphs $D$ such that $\langle D\rangle$ has a specific semigroup property. For instance, in [25] those digraphs $D$ such that $\langle D\rangle$ is regular are classified; and in [5] those $D$ where $\langle D\rangle$ is a band are classified. In [25, 26], necessary and sufficient conditions on digraphs $D$ and $D^{\prime}$ are given so that $\langle D\rangle=\left\langle D^{\prime}\right\rangle$ or $\langle D\rangle \cong\left\langle D^{\prime}\right\rangle$, respectively. In this paper, we continue in this direction, by classifying those digraphs $D$ for which the semigroup $\langle D\rangle$ has one of a variety of properties.

The paper is organised as follows. In Section 2 we review some relevant terminology and basic results about digraphs and semigroups. In Section 3 we investigate the presence of transformations with long cycles in arc-generated semigroups and classify those arc-generated semigroups that are $\mathcal{H}$-trivial. It is possible that Proposition 3.6 and the converse of Proposition 3.12 in Section 3 can be proved using 
Theorem 1(5)(b) and Lemma 15 from [11]. Our proofs were produced independently of the results in [11], and are relatively concise and self-contained, and so we have included the proofs for the sake of completeness. Arc-generated semigroups that are $\mathcal{L}$-, $\mathcal{R}$ - or $\mathcal{J}$-trivial are classified in Section 4 . Further classes of arc-generated semigroups (including bands, completely regular semigroups, inverse semigroups, semilattices and commutative semigroups) are classified in Section 5. Finally, properties related to left and right zeros are classified in Section 6, which among other things, allows us to classify those arc-generated semigroups that are rectangular bands, simple, 0 -simple, or congruence-free.

Many of the results in Sections 4, 5, and 6 were suggested by initial computational experiments conducted using the Semigroups package [17] for GAP [22].

\section{Preliminaries}

2.1. Digraphs. In this subsection, we review some terminology and basic results on digraphs. We refer the reader to [2] for an authoritative account of digraphs.

Unless otherwise stated, the vertex set of a digraph will be $[n]$ for some $n \in \mathbb{N}$.

The in-degree of a vertex $v$ in a digraph $D$ is the number of arcs of the form $(u, v)$ in $D$; similarly, the out-degree of $v$ is the number of $\operatorname{arcs}$ of the form $(v, u)$ in $D$. A vertex $v$ in a digraph $D$ is called a sink if the out-degree of $v$ is 0 . A vertex is isolated if it has no incoming or outgoing arcs.

If $D=(V, A)$ is a digraph, and $U$ is subset of the vertices $V$ of $D$, then the subdigraph of $D$ induced by $U$ is the digraph with vertices $U$ and $\operatorname{arcs} A \cap(U \times U)$. In general, a subdigraph of $D=(V, A)$ is any digraph $D^{\prime}=\left(V^{\prime}, A^{\prime}\right)$ with $V^{\prime} \subseteq V$ and $A^{\prime} \subseteq A \cap\left(V^{\prime} \times V^{\prime}\right)$.

If $D=(V, A)$ is a digraph, and $\varepsilon$ is an equivalence relation on $V$, then the quotient digraph $D / \varepsilon$ is defined as follows. The vertex set is the set of all $\varepsilon$-classes of $V$, and if $W, U$ are $\varepsilon$-classes, then $D / \varepsilon$ has the arc $(W, U)$ if and only if $W \neq U$ and $D$ has an $\operatorname{arc}(w, u)$ for some $w \in W$ and $u \in U$.

A walk in a digraph is a finite sequence $\left(v_{0}, v_{1}, \ldots, v_{r}\right), r \geqslant 1$, of vertices such that $\left(v_{i}, v_{i+1}\right)$ is an arc for all $i \in\{0, \ldots, r-1\}$; the length of this walk is $r$. A path is a walk where all vertices are distinct. A cycle in a digraph is a walk where $v_{0}=v_{r}$ and all other vertices are distinct. A digraph is called acyclic if it has no cycles.

A graph $G$ is defined to be a digraph where $(u, v)$ is an arc if and only if $(v, u)$ is an $\operatorname{arc}$ in $G$. We refer to the pair of arcs above as the edge $\{u, v\}$. Vertices $u$ and $v$ of a graph $G$ are adjacent if $\{u, v\}$ is an edge of $G$.

An induced subdigraph of a graph, is also a graph, which we refer to as an induced subgraph. A spanning subgraph (as opposed to an induced subgraph) of a graph $G=$ $(V, A)$ is any graph $H=(V, B)$ where $B \subseteq A$.

The degree of a vertex in a graph is its in-degree, which equals its out-degree. If $u$ and $v$ are vertices of a graph $G$, then the distance from $u$ to $v$ is the length of a shortest path from $u$ to $v$, if such a path exists.

If $G$ and $H$ are graphs, then $H$ is a minor of $G$ if $H$ can be obtained by successively deleting vertices, deleting edges, or contracting edges of $G$ (where contracting an edge corresponds to deleting it and then identifying its end vertices).

If $v$ is a vertex of a digraph $D$, then the strong component of $v$ is the induced subdigraph of $D$ with vertices $v$ and all $u$ such that there is a path from $u$ to $v$ and from $v$ to $u$. Every digraph is partitioned by its strong components, and the quotient of a digraph by its strong components is acyclic. If $D$ only has one strong component, then it is strongly connected. A terminal component of a digraph $D$ is a strong component $C$ such that $(u, v)$ is not an arc in $D$ for all $u \in C, v \notin C$. 
Alternatively, $C$ is terminal if it is a sink in the quotient of a digraph by its strong components. A strong component of a digraph is trivial if it only has one vertex.

The underlying graph of a digraph $D$ is the graph with an edge $\{u, v\}$ for each arc $(u, v)$ of $D$. The component of $v$ is the induced subdigraph of $D$ with vertices $u$ such that there is a path from $u$ to $v$ in the underlying graph of $D$. Every digraph is partitioned by its components; we say the digraph is connected if it only has one component.

A graph $G$ is separable if it can be decomposed into two connected induced subgraphs $G_{1}$ and $G_{2}$ with exactly one vertex in common, where all edge of $G$ s belong to either $G_{1}$ or $G_{2}$; a graph is non-separable if $G$ admits no such decomposition. A block of a graph is an induced subgraph that is non-separable and is maximal with respect to this property.

A graph $G$ is bipartite if it can be decomposed into two subgraphs $G_{1}$ and $G_{2}$ such that every edge connects a vertex from $G_{1}$ with a vertex from $G_{2}$. A graph is odd bipartite if it is bipartite and it has an odd number of vertices.

We denote by $K_{n}$ the complete graph with vertices $[n]$ and edges $\{u, v\}$ for all distinct $u, v \in[n]$; by $K_{k, 1}$ the star graph with vertices $\{1, \ldots, k+1\}$ and edges $\{i, k+1\}$ for all $i \in\{1, \ldots, k\}$. We denote by $P_{n}$ the path graph, or simply path if there is no ambiguity, with vertices $[n]$ and edges $\{i, i+1\}$ for all $i \in\{1, \ldots, n-1\}$. We denote by $C_{n}$ the cycle graph with vertices $\{1, \ldots, n\}$ and edges $\{1, n\}$ and $\{i, i+1\}$ for all $i \in\{1, \ldots, n-1\}$.

2.2. SEmigroups AND MONOIDS. In this subsection, we review some terminology about semigroups. We refer the reader to [14] and [7] for further background material about semigroups.

A semigroup is a set with an associative binary operation. A monoid is a semigroup $S$ with an identity: i.e. an element $e \in S$ such that $e s=s e=s$ for all $s \in S$. If $S$ is a semigroup, then $s \in S$ is an idempotent if $s^{2}=s$. If $S$ is a semigroup without identity, then we denote by $S^{1}$ the monoid obtained by adjoining an identity $1_{S} \notin S$ to $S$; if $S$ is a monoid, then $S^{1}=S$. A semigroup $S$ is regular if for all $x \in S$ there exists $y \in S$ such that $x y x=x$. A subsemigroup of a semigroup $S$ is a subset $T$ of $S$ that is also a semigroup with the same operation as $S$; denoted $T \leqslant S$.

A congruence on a semigroup $S$ is an equivalence relation $\varepsilon$ on $S$ for which $(a, b) \in \varepsilon$ and $(c, d) \in \varepsilon$ imply $(a c, b d) \in \varepsilon$ for all $a, b, c, d \in S$. A semigroup $S$ is congruence-free if the only congruences on $S$ are the universal and trivial relations.

Let $S$ be a semigroup and let $x, y \in S$ be arbitrary. We say that $x$ and $y$ are $\mathcal{L}$ related if the principal left ideals generated by $x$ and $y$ in $S$ are equal; in other words, if $S^{1} x=\left\{s x: s \in S^{1}\right\}=S^{1} y=\left\{s y: s \in S^{1}\right\}$. We write $x \mathcal{L} y$ to denote that $x$ and $y$ are $\mathcal{L}$-related.

Green's $\mathcal{R}$-relation is defined dually to Green's $\mathcal{L}$-relation; Green's $\mathcal{H}$-relation is the meet, in the lattice of equivalence relations on $S$, of $\mathcal{L}$ and $\mathcal{R}$. Green's $\mathcal{J}$-relation is defined so that $x, y \in S$ are $\mathcal{J}$-related if $S^{1} x S^{1}=S^{1} y S^{1}$. We will refer to the equivalence classes as $\mathcal{K}$-classes where $\mathcal{K}$ is any of $\mathcal{R}, \mathcal{L}, \mathcal{H}$, or $\mathcal{J}$. We write $x \mathcal{K} y$ to indicate $(x, y) \in \mathcal{K}$, where $\mathcal{K}$ is any of $\mathcal{R}, \mathcal{L}, \mathcal{H}$, or $\mathcal{J}$.

We denote by $\operatorname{Tran}_{n}$ the monoid consisting of all of the transformations of degree $n$ where $n \in \mathbb{N}$; called the full transformation monoid. This monoid plays the same role in semigroup theory as the symmetric group does in group theory, in that every finite semigroup is isomorphic to a subsemigroup of some $\operatorname{Tran}_{n}$. Green's relations on $\operatorname{Tran}_{n}$ can be described in terms of the following natural parameters associated to transformations. The image of a transformation $\alpha \in \operatorname{Tran}_{n}$ is the set

$$
\operatorname{im}(\alpha)=\{x \alpha: x \in\{1, \ldots, n\}\}
$$


the kernel of $\alpha$ is the equivalence relation

$$
\operatorname{ker}(\alpha)=\{(x, y) \in\{1, \ldots, n\} \times\{1, \ldots, n\}: x \alpha=y \alpha\}
$$

and the rank of $\alpha$ is

$$
\operatorname{rk}(\alpha)=|\operatorname{im}(\alpha)|
$$

It is well-known that two elements of $\operatorname{Tran}_{n}$ are $\mathcal{R}-, \mathcal{L}$ - or $\mathcal{J}$-related if and only if they have the same kernel, image or rank, respectively; see [14, Exercise 2.6.16].

A semigroup is aperiodic if all of its subgroups are trivial. A semigroup is $\mathcal{K}$-trivial for $\mathcal{K} \in\{\mathcal{R}, \mathcal{L}, \mathcal{H}, \mathcal{J}\}$, if $x \mathcal{K} y$ implies $x=y$.

2.3. Arc-Generated Semigroups. We now characterise some basic semigroup theoretic properties of $\langle D\rangle$ in terms of digraph theoretic properties of $D$.

Suppose that $D$ is a digraph with vertex set $V$, that $v \in V$ is an isolated vertex, and that $D^{\prime}$ is the subdigraph of $D$ induced by $V \backslash\{v\}$. Then it is clear that the arc-generated semigroups $\langle D\rangle$ and $\left\langle D^{\prime}\right\rangle$ are isomorphic. So, we may assume without loss of generality, where appropriate and if it is convenient, that a digraph $D$ has no isolated vertices.

The following proposition will allow us to only consider connected digraphs in some cases; its proof is trivial and is omitted.

Proposition 2.1. Let $D$ be a digraph with components $D_{1}, D_{2}, \ldots, D_{k}$ and no isolated vertices. Then $\langle D\rangle$ is isomorphic to $\left\langle D_{1}\right\rangle^{1} \times \cdots \times\left\langle D_{k}\right\rangle^{1} \backslash\left\{\left(1_{\left\langle D_{1}\right\rangle}, \ldots, 1_{\left\langle D_{k}\right\rangle}\right)\right\}$.

The next result is also trivial.

Proposition 2.2. Let $D$ be a digraph. Then the following are equivalent:

(1) $\langle D\rangle$ is trivial;

(2) $\langle D\rangle$ is a group;

(3) $\langle D\rangle$ has a unique $\mathcal{H}$-class;

(4) D has only one arc.

The semigroup $\langle D\rangle$ can contain arcs that are not present in $D$. It was shown in $[25$, Lemma 2.3] that the set of $\operatorname{arcs}$ in $\langle D\rangle$ is

$$
\{(a \rightarrow b):(a \rightarrow b) \in D \text { or }(b \rightarrow a) \text { belongs to a cycle of } D\} .
$$

The closure of $D$, denoted $\bar{D}$, is the digraph on $[n]$ with the set of arcs as above; it is clear that $\langle\bar{D}\rangle=\langle D\rangle$. By construction, $\bar{D}=D$ if and only if every strong component $C$ of $D$ is a graph. We say that $D$ is closed if $\bar{D}=D$.

\section{CYCLIC PROPERTIES}

A cycle of length $k$ in $\alpha \in \operatorname{Tran}_{n}$ is a sequence of distinct points $a_{0}, a_{1}, \ldots, a_{k-1} \in[n]$ such that $a_{i} \alpha=a_{i+1}$ for all $i$, where the indices are computed modulo $k$. This section is concerned with cycles of transformations in an arc-generated semigroup. In particular, we are interested in the presence of long cycles. As mentioned in the introduction, the results in this section are related to those in [11], where the authors describe the structure and actions of the maximal subgroups of any arc-generated semigroup $\langle D\rangle$ in terms of properties of $D$. It is possible that Proposition 3.6 and the converse of Proposition 3.12 could be proved using Theorem 1(5)(b) and Lemma 15 from [11]. However, determining the properties of the specific digraphs in Propositions 3.6 and 3.12 required to apply the results in [11] is non-trivial, and requires the notation and terminology used in [11]. Since the proofs presented in this section are self-contained, and relatively concise, and were found independently of [11], we have opted not to use the results of [11]. 
One application of the results in this section is a classification of the digraphs $D$ such that $\langle D\rangle$ is $\mathcal{H}$-trivial. In particular, we shall prove the following result.

Proposition 3.1. Let $D$ be a digraph. Then $\langle D\rangle$ is $\mathcal{H}$-trivial if and only if all the strong components of $D$ are paths or isolated vertices.

3.1. Preliminary Results. The length of a longest cycle of $\alpha$ is denoted as $l(\alpha)$ and for a digraph $D$ we write

$$
l(D)=\max \{l(\alpha): \alpha \in\langle D\rangle\} .
$$

LEMMA 3.2. Let $D$ be a digraph. Then the following are equivalent:

(i) $l(D)=1$;

(ii) $\langle D\rangle$ is aperiodic;

(iii) $\langle D\rangle$ is $\mathcal{H}$-trivial.

Proof. Conditions (ii) and (iii) are equivalent for any finite semigroup; see [18, Proposition 4.2 .

(i) $\Rightarrow$ (ii). We prove the contrapositive. Suppose $\alpha \in\langle D\rangle$ belongs to a non-trivial subgroup and that $\alpha$ is not an idempotent. Then the restriction of $\alpha$ to $\operatorname{im}(\alpha)$ is a non-trivial permutation, so $l(\alpha) \geqslant 2$.

(ii) $\Rightarrow$ (i). Again, we prove the contrapositive. Suppose $\alpha \in\langle D\rangle$ has a cycle of length $k \geqslant 2$, say $a_{0}, a_{1}, \ldots, a_{k-1}$. Choose $r \geqslant 1$ such that $\alpha^{r}$ is an idempotent, and let $H$ be the $\mathcal{H}$-class of $\alpha^{r}$. Then $H$ is a group, and $H=\left\{\alpha^{s}: s \geqslant r\right\}$. But $\alpha^{r}$ and $\alpha^{r+1}$ are distinct elements of $H$, since $a_{0} \alpha^{r}=a_{0} \neq a_{1}=a_{0} \alpha^{r+1}$.

Since $\langle D\rangle=\langle\bar{D}\rangle$ for any digraph $D$, we clearly have $l(D)=l(\bar{D})$. Thus, when studying $l(D)$, we can assume without loss of generality that $D$ is closed. If $\alpha \in\langle D\rangle$, then any cycle of $\alpha$ belongs entirely to a strong component of $D$. Therefore, if $D$ has strong components $S_{1}, \ldots, S_{r}$, then

$$
l(D)=\max \left\{l\left(S_{1}\right), \ldots, l\left(S_{r}\right)\right\} .
$$

Thus, in this section we may assume without loss of generality, if it is convenient, that $D$ is a connected graph.

LEMma 3.3. For the cycle graph $C_{n}, n \geqslant 3$, we have $l\left(C_{n}\right)=n-1$.

Proof. Clearly, for any digraph $G$ on $n$ vertices, $l(G) \leqslant n-1$. Conversely,

$$
(n-1 \rightarrow n)(n-2 \rightarrow n-1) \cdots(1 \rightarrow 2)(n \rightarrow 1) \in\left\langle C_{n}\right\rangle
$$

has the cycle $1,2, \ldots, n-1$.

If $G^{\prime}$ is a subgraph of $G$, then $\left\langle G^{\prime}\right\rangle \leqslant\langle G\rangle$, and so $l\left(G^{\prime}\right) \leqslant l(G)$, which will allow us to isolate subgraphs of $G$ in order to obtain lower bounds on $l(G)$. In the next lemma, we extend this result to graph minors.

LEMMA 3.4. If $G$ is a graph and $H$ is a minor of $G$, then $l(H) \leqslant l(G)$.

Proof. For any $k \leqslant n$ and $S \leqslant \operatorname{Tran}_{k}$ and any $T \leqslant \operatorname{Tran}_{n}$, we write $S \preceq T$ if, relabelling the vertices of $\{1, \ldots, k\}$ if necessary, for any $\alpha \in S$, there exists $\beta \in T$ such that $v \beta=v \alpha$ for all $v \in\{1, \ldots, k\}$. It is clear that if $\langle G\rangle \preceq\langle H\rangle$, then $l(G) \leqslant l(H)$. Hence it suffices to show that $\langle H\rangle \preceq\langle G\rangle$.

This clearly holds if $H$ is obtained from $G$ by deleting an edge or a vertex. Suppose that $H$ is obtained from $G$ by contracting the edge $\{n-1, n\}$. Let $B$ be the set of vertices that are adjacent to $n$ but not to $n-1$ in $G$.

Let $\alpha \in\langle H\rangle$ be arbitrary. Then there exist arcs $\beta_{1}, \ldots, \beta_{k} \in H$ such that $\alpha=$ $\beta_{1} \beta_{2} \cdots \beta_{k}$. If $\beta_{i}=(b \rightarrow n-1)$ for some $b \in B$, then we replace $\beta_{i}$ in the product 
for $\alpha$ by $(b \rightarrow n)(n \rightarrow n-1)$. Similarly, we replace any arc $(n-1 \rightarrow b), b \in B$, by $(n-1 \rightarrow n)(n \rightarrow b)$. If $\beta \in\langle G\rangle$ denotes this modified product, then $v \beta=v \alpha$ for all $v \in\{1, \ldots, n-1\}$, and so $\langle H\rangle \preceq\langle G\rangle$.

\section{LEMmA 3.5. Let $G$ be a graph. Then the following hold:}

(i) if $G$ has a vertex of degree $k$, then $l(G) \geqslant k-1$;

(ii) if $G$ contains a subgraph that is a tree with $k$ leaves, then $l(G) \geqslant k-1$;

(iii) if $G$ is connected and $t$ is the number of vertices of degree not equal to 2 , then

$$
l(G) \geqslant \frac{1}{4}(t-2)+1 .
$$

Proof. (i). For distinct vertices $u$ and $v$ of the star graph $K_{k, 1}$ such that $u, v \neq k+1$, we write $(u \rightsquigarrow v)=(u \rightarrow k+1)(k+1 \rightarrow v)$. Then

$$
(k-1 \rightsquigarrow k)(k-2 \rightsquigarrow k-1) \cdots(1 \rightsquigarrow 2)(k \rightsquigarrow 1) \in\left\langle K_{k, 1}\right\rangle
$$

has the cycle $1,2, \ldots, k-1$. The result now follows from Lemma 3.4.

(ii). If $T$ is a tree with $k$ leaves, then $K_{k, 1}$ is a minor of $T$, so the result follows from Lemma 3.4 and part (i).

(iii). By [3], any graph with $t$ vertices of degree not equal to 2 contains a spanning tree with at least $\frac{1}{4}(t-2)+2$ leaves.

The next result concerns connected graphs that can be decomposed into two connected induced subgraphs with a path connecting them. With this in mind, we introduce a construction based on paths. Let $L$ and $R$ be two connected graphs on $m$ and $s$ vertices, respectively, where $m \leqslant s$, and let $P$ be a path with $q$ vertices. Let $L \oplus_{q} R$ denote the graph obtained by adding an edge between an endpoint of $P$ and a vertex of $L$ of degree not equal to 1 , and an edge between the other endpoint of $P$ to a vertex of $R$ of degree not equal to 1 . Even though this definition depends on the choice of attachment vertices, we will omit them in the notation, for our purpose is to derive results that do not depend on them, apart from the fact that they do not have degree 1 in $L$ and $R$. We remark that $m, s \neq 2$, since the only connected graph on two vertices is $K_{2}$, whose vertices both have degree 1 . However, it is possible to have $m=1$ or $s=1$.

The vertices of $R$ are denoted as $r_{1}, \ldots, r_{s}$, where they are sorted in weakly increasing order of distance to the path $P$. In particular, $r_{1}$ is attached to $P$, and $r_{2}$ and $r_{3}$ are neighbours of $r_{1}$ if $s \neq 1$. A similar notation is used for $L$; in particular $l_{1}$ is attached to $P$. Write $L^{*}=L \backslash\left\{l_{1}\right\}, R^{*}=R \backslash\left\{r_{1}\right\}, P^{*}=P \cup\left\{l_{1}, r_{1}\right\}$, and order the elements of the path as $p_{1}, \ldots, p_{q}$ so that $p_{1}$ is adjacent to $l_{1}$, and $p_{q}$ is adjacent to $r_{1}$; finally, we also write $l_{1}=p_{0}$ and $r_{1}=p_{q+1}$. For instance, the graph $K_{3,1} \oplus_{4} C_{4}$ is illustrated in Figure 2.

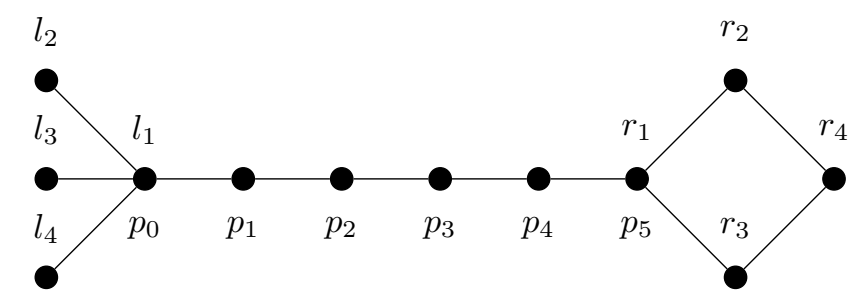

Figure 2. The graph $K_{3,1} \oplus_{4} C_{4}$. 
Proposition 3.6 (cf. Theorem 1(5)(b) in [11]). With the above notation, if $q \geqslant s$, then

$$
l\left(L \oplus_{q} R\right)= \begin{cases}1 & \text { if } m=s=1 \\ s-1 & \text { if } m=1, s \geqslant 3 \\ m+s-3 & \text { otherwise. }\end{cases}
$$

Proof. We write $G=L \oplus_{q} R$. If $m=s=1$, then $G=P_{n}$ where $n=q+2$, and hence $\left\langle P_{n}\right\rangle$ is the semigroup of order-preserving transformations [1], which is aperiodic. Otherwise, we have $s \geqslant 3$ and we view the result as two matching upper and lower bounds on $l(G)$.

LOwER BOUND. Throughout this part of the proof, if $u, w_{1}, \ldots, w_{t}, v$ is a path in $G$, then we define

$$
(u \rightsquigarrow v)=\left(u \rightarrow w_{1}\right)\left(w_{1} \rightarrow w_{2}\right) \cdots\left(w_{t-1} \rightarrow w_{t}\right)\left(w_{t} \rightarrow v\right) \in\langle G\rangle .
$$

Since this transformation depends on the choice of path, we will always specify the path.

CASE 1: $m=1$. We will show that there exists $\alpha \in\langle G\rangle$ containing the cycle $r_{2}, \ldots, r_{s}$.

For each $3 \leqslant i \leqslant s$, we choose $r_{i}^{\prime} \in\left\{r_{2}, r_{3}\right\}$ such that there is a shortest-length path from $r_{1}$ to $r_{i}$ that avoids $r_{i}^{\prime}$. We also define

- $\left(r_{2} \rightsquigarrow p_{2}\right)$ to follow the path $r_{2}, r_{1}, p_{q}, \ldots, p_{2}$,

- $\left(p_{j-1} \rightsquigarrow r_{j}\right)$ to follow a shortest-length path avoiding $r_{j}^{\prime}$ and $\left(r_{j} \rightsquigarrow p_{j}\right)$ to follow the reverse of such a path (but omitting the last edge) for all $3 \leqslant j \leqslant s$,

- $\left(r_{j}^{\prime} \rightsquigarrow r_{j-1}^{\prime}\right)=\left(r_{j}^{\prime} \rightarrow r_{1}\right)\left(r_{1} \rightarrow r_{j-1}^{\prime}\right)$ for all $4 \leqslant j \leqslant s$, even if $r_{j}^{\prime}=r_{j-1}^{\prime}$,

- $\left(r_{s} \rightsquigarrow r_{s}^{\prime}\right)$ to follow any path avoiding vertices from $P$.

It is straightforward to verify that

$$
\alpha=\left[\prod_{i=2}^{s-1}\left(r_{i} \rightsquigarrow p_{i}\right)\right] \cdot\left(r_{s} \rightsquigarrow r_{s}^{\prime}\right) \cdot\left[\prod_{j=s}^{4}\left(p_{j-1} \rightsquigarrow r_{j}\right)\left(r_{j}^{\prime} \rightsquigarrow r_{j-1}^{\prime}\right)\right] \cdot\left(p_{2} \rightsquigarrow r_{3}\right) \in\langle G\rangle
$$

contains the cycle $r_{2}, \ldots, r_{s}$, as required (the second product is computed in descending order of the indices). We also note that $l_{1} \alpha=l_{1}$.

CASE 2: $m \geqslant 3$. As in Case 1 , we may use $K_{1} \oplus_{q} R \subseteq G$ to create $\alpha \in\langle G\rangle$ containing the cycle $r_{2}, \ldots, r_{s}$ and such that $l_{i} \alpha=l_{i}$ for all $i$. Similarly, we may use $L \oplus_{q} K_{1} \subseteq G$ to create $\beta \in\langle G\rangle$ containing the cycle $l_{2}, \ldots, l_{m}$ and such that $r_{i} \beta=r_{i}$ for all $i$. If $\left(r_{2} \rightsquigarrow l_{3}\right)$ and $\left(l_{2} \rightsquigarrow r_{2}\right)$ follow the unique shortest paths, then $\gamma=\alpha \beta\left(r_{2} \rightsquigarrow l_{3}\right)\left(l_{2} \rightsquigarrow\right.$ $\left.r_{2}\right) \in\langle G\rangle$ contains the cycle $l_{3}, \ldots, l_{m}, r_{2}, \ldots, r_{s}$.

UPPER BOUND. Since $l\left(G_{1}\right) \leqslant l\left(G_{2}\right)$ if $G_{1}$ is a subgraph of $G_{2}$, we assume without loss of generality that $G=K_{m} \oplus_{q} K_{s}$. We define a pre-order $\preceq$ on the vertices of $G$ such that $a \preceq b$ if $a \in L^{*}$, or $b \in R^{*}$, or $a=p_{i}$ and $b=p_{j}$ for some $0 \leqslant i \leqslant j \leqslant q+1$. If $a \preceq b$ and $b \npreceq a$, then we write $a \prec b$ or $b \succ a$. We note that $b \npreceq a$ implies $a \preceq b$. We define the sets

$$
a^{+}=\{b \in[n]: b \succ a\} \cup\{a\} \quad \text { and } \quad a^{-}=\{b \in[n]: a \succ b\} \cup\{a\} .
$$

For the remainder of the proof, we fix some $\gamma \in\langle G\rangle$, and we write $\gamma=\epsilon_{1} \cdots \epsilon_{k}$, where $\epsilon_{1}, \ldots, \epsilon_{k}$ are arcs in $G$. We also define $\gamma_{0}=$ id and $\gamma_{i}=\epsilon_{1} \cdots \epsilon_{i}$ for all $1 \leqslant i \leqslant k$.

CASE 1: $m=1$. We require the following claim.

Claim 3.7. Suppose that $m=1$. If there are vertices $u$ and $v$ in $G$ such that $u \preceq v$ and $u \gamma \succ v \gamma$, then $\gamma \in \alpha\langle G\rangle$ for some $\alpha \in\langle G\rangle \cup\{\mathrm{id}\}$ such that $u^{+} \alpha \subseteq R^{*}$. 
Proof. If $u \in R^{*}$, then $u^{+}=\{u\}$ and so $\alpha=$ id has the required properties. Suppose that $u \notin R^{*}$. Since $u \gamma \succ v \gamma$, there exists $i$ such that $u \gamma_{i} \in R^{*}$. If $j$ is the least such value, then $u \gamma_{j-1}=r_{1}, u^{+} \gamma_{j-1} \subseteq R$, and $\epsilon_{j}=\left(r_{1} \rightarrow r_{a}\right)$ for some $r_{a} \in R^{*}$. If we set $\alpha=\gamma_{j}$, then $u^{+} \alpha=\left(u^{+} \gamma_{j-1}\right) \epsilon_{j} \subseteq R^{*}$.

Seeking a contradiction, suppose that $\gamma$ has a cycle of length $c$, where $c \geqslant s$, and let $C=\left\{u_{1}, \ldots, u_{c}\right\}$ be such a cycle, where $u_{1} \preceq \cdots \preceq u_{c}$. It is not necessarily the case that $u_{i} \gamma=u_{i+1}$. Since $|C|=c>s-1=\left|R^{*}\right|, C$ is not contained in $R^{*}$ and so $u_{1} \prec u_{i}$ for all $i \neq 1$. This gives $C \subseteq u_{1}^{+}$. Let $j$ be such that $u_{j} \gamma=u_{1}$. Then $u_{1} \prec u_{j}$ but $u_{1} \gamma \succ u_{j} \gamma$. So by Claim 3.7, $c=|C \gamma| \leqslant\left|u_{1}^{+} \gamma\right| \leqslant\left|R^{*}\right|=s-1$, which is the desired contradiction.

CASE 2: $m \geqslant 3$. For the sake of obtaining a contradiction, suppose that $\gamma$ has a cycle of length at least $m+s-2$. Let $C=\left\{u_{1}, \ldots, u_{c}\right\}$ be such a cycle, sorted so that $u_{1} \preceq \cdots \preceq u_{c}$. Again it is not necessarily the case that $u_{i} \gamma=u_{i+1}$. We note that $u_{c} \notin L$ (since $\left.c \geqslant m+1\right)$ and $u_{1} \notin R$ (since $c \geqslant s+1$ ), whence $u_{c} \succ u_{1}$.

We say that a vertex $v$ of $G$ is of $L$-type if there is $\beta \in\langle G\rangle \cup\{\mathrm{id}\}$ such that $\gamma \in \beta\langle G\rangle$ and $v^{-} \beta \subseteq L^{*}$. Similarly, we say that $v$ is of $R$-type if there is $\alpha \in\langle G\rangle \cup\{$ id $\}$ such that $\gamma \in \alpha\langle G\rangle$ and $v^{+} \alpha \subseteq R^{*}$.

Claim 3.8. Suppose that $m \geqslant 3$. If there are vertices $u$ and $v$ in $G$ such that $u \preceq v$ and $u \gamma \succ v \gamma$, then either $u$ is of R-type, or $v$ is of L-type.

Proof. As in the proof of Claim 3.7, if $u \in R^{*}$, then $\alpha=$ id witnesses that $u$ is of $R$-type. Similarly, if $v \in L^{*}$ then $\beta=$ id shows that $v$ is of $L$-type.

Suppose that $u \notin R^{*}$ and $v \notin L^{*}$. As before, for some $i \in\{1, \ldots, k\}, u \gamma_{i}$ and $v \gamma_{i}$ both belong to $L^{*}$ or $R^{*}$. Suppose that both $u \gamma_{i}$ and $v \gamma_{i}$ belong to $R^{*}$ before they both belong to $L^{*}$; the case when they both first belong to $L^{*}$ is symmetric. Let $i$ be the least value such that $u \gamma_{i}, v \gamma_{i} \in R^{*}$. Then for all $j<i, u \gamma_{j} \preceq v \gamma_{j}$ and either $u \gamma_{j} \notin R^{*}$ or $v \gamma_{j} \notin R^{*}$. If $u \gamma_{j} \in R^{*}$ for some $j<i$, then since $u \gamma_{j} \preceq v \gamma_{j}$, it follows that $v \gamma_{j} \in R^{*}$. Hence $i$ is the least value such that $u \gamma_{i} \in R^{*}$.

We will show that $u \gamma_{j} \notin L^{*}$ for all $0 \leqslant j<i$. Seeking a contradiction, suppose that $u \gamma_{j} \in L^{*}$ for some $0 \leqslant j<i$, and let $b=\max \left\{j: j<i, u \gamma_{j} \in L^{*}\right\}$. The vertices $[n]$ of $G$ can be partitioned into two parts:

$$
A=\left(\left\{u \gamma_{b}\right\} \cup P^{*} \cup R^{*}\right) \gamma_{b}^{-1} \quad \text { and } \quad B=\left(L^{*} \backslash\left\{u \gamma_{b}\right\}\right) \gamma_{b}^{-1} .
$$

Let $x \in A$. If $x \gamma_{b}=u \gamma_{b}$, then $x \gamma_{i}=u \gamma_{i} \in R^{*}$. Otherwise, $u \gamma_{b} \prec x \gamma_{b}$. By maximality of $b$ and minimality of $i$, we have $\epsilon_{b+1}=\left(u \gamma_{b} \rightarrow l_{1}\right), \epsilon_{i}=\left(r_{1} \rightarrow u \gamma_{i}\right), u \gamma_{j} \in P^{*}$ for all $b<j<i$, and $u \gamma_{j} \preceq x \gamma_{j}$ for all $b<j<i$. It follows that $x \gamma_{i} \in R^{*}$. Therefore, $A \gamma_{i} \subseteq R^{*}$, and so

$$
c \leqslant \operatorname{rk}(\gamma) \leqslant\left|B \gamma_{b}\right|+\left|A \gamma_{i}\right| \leqslant(m-2)+(s-1)=m+s-3,
$$

which contradicts the fact that $c>m+s-3$.

We conclude that $u \gamma_{j} \in P^{*}$ for all $j<i$, and by the argument concluding the proof of Claim 3.7, we obtain $u^{+} \gamma_{i} \subseteq R^{*}$, so that $u$ is of $R$-type.

Claim 3.9. There exist $u, v \in C$ such that $u \preceq v, u_{c} \preceq v$, and $u \gamma \succ v \gamma$. There also exist $u^{\prime}, v^{\prime} \in C$ such that $u^{\prime} \preceq v^{\prime}, u^{\prime} \preceq u_{1}$, and $u^{\prime} \gamma \succ v^{\prime} \gamma$.

Proof. If $u_{c} \in R^{*}$, then since $C$ intersects $R^{*}$ but is not contained in $R^{*}$, there exist $u, v \in C$ such that $u \notin R^{*}, u \gamma \in R^{*}, v \in R^{*}$, and $v \gamma \notin R^{*}$. Then $u$ and $v$ have the required properties.

If $u_{c} \notin R^{*}$, then $u_{c} \succ u_{i}$ for all $1 \leqslant i \leqslant c-1$. In particular, if $u_{c}=u_{j} \gamma$, then $u=u_{j}$ and $v=u_{c}$ have the required properties.

The proof of the existence of $u^{\prime}$ and $v^{\prime}$ is symmetrical. 
We now write $X=\left\{u_{i} \in C: \exists j, u_{i} \preceq u_{j}, u_{i} \gamma \succ u_{j} \gamma\right\}$, and note that $X \neq \varnothing$ by Claim 3.9. We enumerate $X=\left\{x_{1}, \ldots, x_{d}\right\}$ such that $x_{1} \preceq \cdots \preceq x_{d}$. For each $1 \leqslant i \leqslant d$, let $y_{i}$ be an element of $C$ such that $x_{i} \preceq y_{i}$ and $x_{i} \gamma \succ y_{i} \gamma$; we also assume that $y_{i}$ is maximal with respect to this property: that is, if $v \in C$ is such that $x_{i} \preceq v$ and $x_{i} \gamma \succ v \gamma$, then $v \preceq y_{i}$. Note that $\left\{y_{1}, \ldots, y_{d}\right\}$ is not necessarily sorted according to the pre-order. If $y_{M}$ is a maximal element of $\left\{y_{1}, \ldots, y_{d}\right\}$ with respect to $\preceq$, then Claim 3.9 indicates that $u_{c} \preceq y_{M}$. We also have $x_{1} \preceq u_{1}$, since $x_{1} \preceq u^{\prime}$, where $u^{\prime}$ is as in Claim 3.9.

Claim 3.10. There exists $1 \leqslant a<d$ such that $y_{1}, \ldots, y_{a}$ are all of L-type and $x_{a+1}, \ldots, x_{d}$ are all of $R$-type. Moreover, for all $i>a \geqslant j, x_{i} \succ y_{j}$.

Proof. We shall prove a sequence of facts about the set $X$, the last two of which give the claim.

(a) If $x_{i}$ is of $R$-type, and if $x_{i} \preceq y_{j}$, then $y_{j}$ is not of $L$-type.

Suppose to the contrary that we have the following: $x_{i} \preceq y_{j} ; \alpha, \beta \in\langle G\rangle \cup$ $\{$ id $\} ; \gamma \in \alpha\langle G\rangle$ and $\gamma \in \beta\langle G\rangle ; x_{i}^{+} \alpha \subseteq R^{*}$ and $y_{j}^{-} \beta \subseteq L^{*}$. We then have $x_{i} \notin L^{*}$, for otherwise $[n]=L^{*} \cup x_{i}^{+}$and

$$
c \leqslant \operatorname{rk}(\gamma) \leqslant\left|L^{*} \backslash\left\{x_{i}\right\}\right|+\left|x_{i}^{+} \alpha\right| \leqslant(m-2)+(s-1)=m+s-3,
$$

a contradiction. Similarly, we have $y_{j} \notin R^{*}$. Thus

$$
x_{i}, y_{j} \in P^{*}, \quad[n]=x_{i}^{+} \cup y_{j}^{-}, \quad x_{i} \in x_{i}^{+} \cap y_{j}^{-} .
$$

Denoting $S=\left\{w \in y_{j}^{-}: w \gamma=x_{i} \gamma\right\}$, we have

$c \leqslant \operatorname{rk}(\gamma) \leqslant\left|\left(x_{i}^{+} \cup S\right) \gamma\right|+\left|\left(y_{j}^{-} \backslash S\right) \gamma\right|=\left|x_{i}^{+} \gamma\right|+\left|y_{j}^{-} \gamma\right|-1 \leqslant(s-1)+(m-1)-1=m+s-3$, a contradiction.

(b) For every $i, x_{i}$ is of $R$-type if and only if $y_{i}$ is not of $L$-type.

Apply (a) with $i=j$ and combine with Claim 3.8.

(c) If $x_{i}$ is of $R$-type, then so too are $x_{i+1}, \ldots, x_{d}$.

If $x_{i}$ is of $R$-type and $i<j$, then because $x_{i} \preceq x_{j} \preceq y_{j}$, (a) says that $y_{j}$ is not of $L$-type and (b) in turn says that $x_{j}$ is of $R$-type.

(d) $y_{1}$ is of $L$-type and $x_{M}$ is of $R$-type.

We prove that $x_{1}$ is not of $R$-type, which by (b) implies that $y_{1}$ is of $L$-type. Suppose that $x_{1}^{+} \alpha \subseteq R^{*}$ for some $\alpha \in\langle G\rangle \cup\{$ id $\}$ with $\gamma \in \alpha\langle G\rangle$. If $x_{1} \in L^{*}$, then $x_{1}^{+}=\left\{x_{1}\right\} \cup P^{*} \cup R^{*}$ and hence

$c \leqslant \operatorname{rk}(\gamma) \leqslant\left|L^{*} \backslash\left\{x_{1}\right\}\right|+\left|\left(\left\{x_{1}\right\} \cup P^{*} \cup R^{*}\right) \alpha\right| \leqslant(m-2)+(s-1)=m+s-3$,

a contradiction. If $x_{1} \notin L^{*}$, then $x_{1}=u_{1}$ (since $x_{1} \preceq u_{1}$ and $u_{1} \notin R^{*}$ ) and hence $C \subseteq x_{1}^{+}$, so that $c=|C \gamma| \leqslant\left|x_{1}^{+} \alpha\right| \leqslant s-1$, a contradiction. The proof for $x_{M}$ is symmetrical.

(e) There exists $1 \leqslant a<d$ such that $y_{1}, \ldots, y_{a}$ are all of $L$-type and $x_{a+1}, \ldots, x_{d}$ are all of $R$-type.

This follows from combining (b), (c) and (d), with

$$
a=\max \left\{i: y_{1}, \ldots, y_{i} \text { are of } L \text {-type }\right\} \text {. }
$$

(f) For all $i>a \geqslant j, x_{i} \succ y_{j}$.

By (e), $x_{i}$ is of $R$-type, and $y_{j}$ of $L$-type. It follows from (a) that

$$
x_{i} \succ y_{j}
$$

We now partition $C$ into two parts $A$ and $B$ defined by

$$
A=\left\{u \in C: x_{a+1} \succ u\right\} \quad \text { and } \quad B=\left\{v \in C: x_{a+1} \preceq v\right\} .
$$


Note that $A$ and $B$ are both non-empty: for example, $x_{a+1} \in B$ and $y_{a} \in A$. Since $C$ is a cycle of $\gamma, S \gamma \neq S$ for any non-empty proper subset $S$ of $C$. In particular, $A \gamma \neq A$ and $B \gamma \neq B$, and so there exist $u \in A$ and $v \in B$ such that $u \gamma \in B$ and $v \gamma \in A$. It follows that $u \preceq v$ and $u \gamma \succ v \gamma$, and hence $u=x_{j}$ and $v \preceq y_{j}$ for some $j \leqslant a$. But then $x_{a+1} \preceq v \preceq y_{j}$, which contradicts Claim 3.10.

3.2. Classification Results. In this subsection, we give a classification of the connected graphs $G$ for which $l(G)$ is equal to 1,2 or $n-1$. From this, and in light of equation (1), it is easy to deduce such classifications for arbitrary graphs $G$. We also consider the computational complexity of determining whether a given graph $G$ satisfies $l(G) \leqslant k$.

The classification of graphs with $l(G)=1$ or $l(G)=2$ is based on the following family of graphs. The graph $Q_{n}$ for $n \geqslant 3$ is obtained by adding the edge $\{n-2, n\}$ to the path $P_{n}$, so that the three last vertices form a triangle (and indeed $Q_{3}=K_{3}$ ). The graph $R_{n}$ for $n \geqslant 4$ is obtained by removing the edge $\{n-1, n\}$ from $Q_{n}$, so that the last four vertices form the star graph $K_{3,1}$ (and indeed $R_{4}=K_{3,1}$ ). The graphs $Q_{6}$ and $R_{6}$ are illustrated in Figure 3 .
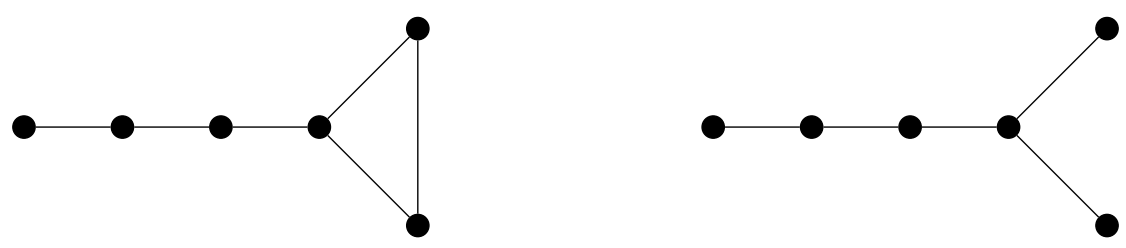

FiguRE 3. The graphs $Q_{6}$ (left) and $R_{6}$ (right).

A number of other graphs, pictured in Figure 4, will feature in the proofs. It can be shown, using GAP [22] for instance, that if $G$ is the bull graph or the E-graph, then $l(G)=3$ and that $l\left(\theta_{0}\right)=6$.
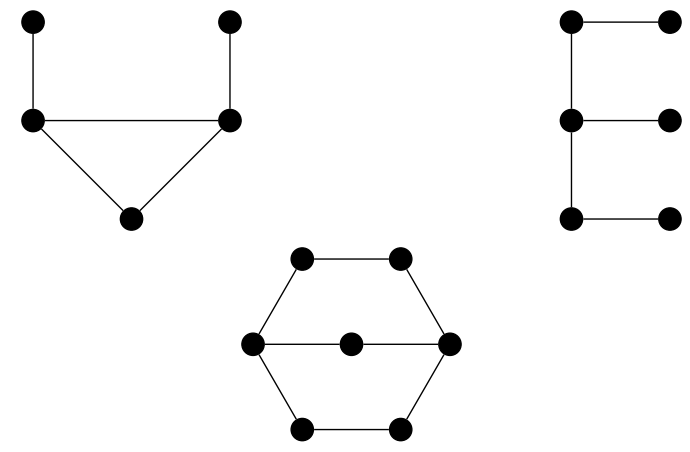

Figure 4. The bull graph (left), E-graph (right) and $\theta_{0}$ graph (below).

Proposition 3.11. Let $G$ be a connected graph. Then $l(G)=1$ if and only if $G$ is a path.

Proof. Since $\left\langle P_{n}\right\rangle$ is the semigroup of order-preserving transformations of $[n]$, it is aperiodic. Conversely, suppose that $l(G)=1$. By Lemmas 3.3, 3.4 and 3.5, $G$ is a tree with maximum degree 2 , in other words, $G$ is a path.

Proposition 3.1 easily follows from Proposition 3.11 and equation (1). 
Proposition 3.12. Let $G$ be a connected graph. Then $l(G)=2$ if and only if $G$ is $Q_{n}(n \geqslant 3)$ or $R_{n}(n \geqslant 4)$.

Proof. Note that $Q_{n}=K_{1} \oplus_{n-4} K_{3}$ and $R_{n}=K_{1} \oplus_{n-4} K_{2,1}$. It follows from Proposition 3.6 that $l\left(Q_{n}\right)=l\left(R_{n}\right)=2$ for $n \geqslant 7$; this can also be verified for $n \leqslant 6$, using GAP [22]. This part of the proof also follows from [11, Lemma 15].

Conversely, suppose that $l(G)=2$; the case $n \leqslant 3$ is easy so let us assume $n \geqslant 4$. By Lemmas 3.3 and 3.4, $G$ does not have any cycle of length 4 or more. By Lemma 3.5, $G$ has no vertices of degree greater than 3 .

Claim 3.13. G has exactly one vertex of degree 3 .

Proof. If $G$ has no vertex of degree 3, then it is a path and $l(G)=1$, or it is a cycle and $l(G)=n-1 \geqslant 3$. Thus, $G$ has a vertex of degree 3 , say $x_{1}$, with neighbours $x_{2}$, $x_{3}$ and $x_{4}$. First, suppose that $x_{2}$ also has degree 3 . If $x_{3}$ and $x_{4}$ are both neighbours of $x_{2}$, then $G$ has the cycle $x_{1}, x_{3}, x_{2}, x_{4}$. If $x_{2}$ is adjacent to $x_{3}$ and to another vertex, say $x_{5}$, then $G$ contains a bull. Thus, $x_{2}$ is not adjacent to either $x_{3}$ or $x_{4}$, and instead is adjacent to $x_{5}$ and $x_{6}$, say, in which case, $G$ contains a tree with leaves $x_{3}, x_{4}, x_{5}$, and $x_{6}$, so Lemma 3.5 (ii) applies. So vertex $x_{2}$ does not have degree $x_{3}$ and, similarly, neither do vertices $x_{3}$ and $x_{4}$. Second, suppose that $G$ contains another vertex of degree 3 , say $u$, that is not a neighbour of $x_{1}$. There is a path from $u$ to $x_{1}$, and we may assume this goes through vertex $x_{2}$; then by contracting the path from $x_{2}$ to $u$, and applying Lemma 3.4, we get back to the first case.

We now split the rest of the proof into two cases. First, if $G$ is a tree, then $G=R_{n}$ or $G$ has the E-graph as a subgraph. The latter case would yield $l(G) \geqslant 3$, hence $G=R_{n}$. Second, if $G$ is not a tree, then $G$ has a triangle, say induced by the vertices $a, b, c$. One of them must be the vertex of degree 3 , say $a$, and the other two have degree 2. Then $G=Q_{n}$.

On the other extreme, we have the following classification. Recall that a graph $G$ is non-separable if for every pair of vertices $u, v \in[n]$, there are at least two vertexdisjoint paths from $u$ to $v$.

Proposition 3.14. Let $G$ be a connected graph. Then $l(G)=n-1$ if and only if $G=K_{2}$ or $G$ is non-separable and not odd bipartite.

Proof. The case $n \leqslant 3$ being easily checked, we assume $n \geqslant 4$ throughout the proof.

Let $G$ be non-separable. Recall the puzzle group $\Gamma_{G}(v)$ from [23], obtained as follows. First of all, create a hole at any vertex $v$. Then repeatedly slide a vertex $a$ into the hole at vertex $b$, where $a$ is adjacent to $b$; this moves the hole to $a$. Whenever the hole goes back to $v$, this yields a permutation of $[n] \backslash\{v\}$. The (abstract) group does not actually depend on $v$. Clearly, creating the hole at $v$ can be done by using any arc $(v \rightarrow u)$ where $u$ is a neighbour of $v$, and then sliding a vertex $a$ to the hole in $b$ is equivalent to using the arc $(a \rightarrow b)$. Therefore, for any initial hole $v$ and any $g \in \Gamma_{G}(v)$ acting on $[n] \backslash\{v\}$, there exists $\alpha \in\langle G\rangle$ such that $u g=u \alpha$ for all $u \neq v$.

$(\Leftarrow)$ We have already noted that $l\left(\theta_{0}\right)=6$, and that $l\left(C_{n}\right)=n-1$. Let $G$ be non-separable and neither a cycle nor the graph $\theta_{0}$. According to [23, Theorem 2], $\Gamma_{G}(v)=\operatorname{Alt}_{n-1}$ if $G$ is bipartite and $\Gamma_{G}(v)=\operatorname{Sym}_{n-1}$ otherwise. Therefore $l(G)=$ $n-1$ if $G$ is non-separable and not odd bipartite, or $l(G) \geqslant n-2$ if $G$ is non-separable and odd bipartite.

$(\Rightarrow)$ We prove the contrapositive. Suppose first that $G$ is non-separable and odd bipartite. To obtain a contradiction, suppose that $\beta \in\langle G\rangle$ has $l(\beta)=n-1$. Due to the form of $\beta$, there exist $u$ and $v$ such that $u \beta=v \beta, u \beta^{-1} \neq \varnothing$ and $v \beta^{-1}=\varnothing$ (note that $\beta$ acts as a cyclic permutation $\pi$ on $[n] \backslash\{v\})$. Since $(v \rightarrow u) \beta=\beta$, we can 
assume that the first arc in any word expressing $\beta$ is $(v \rightarrow u)$. This corresponds to creating a hole in $v$, and then expressing $\pi$ as a member of $\Gamma_{G}(v)$, which is impossible since $\pi$ is an odd permutation while $\Gamma_{G}(v)=\operatorname{Alt}_{n-1}$.

Now suppose that $G$ is separable. So there exist $L, R \subseteq[n]$ and $v \in[n]$ such that $2 \leqslant|L| \leqslant|R|, L \cap R=\{v\}$, and for any edge $\{l, r\}$ of $G$ with $l \in L$ and $r \in R$ we have $v \in\{l, r\}$; see for example [4, Theorems 5.1 and 5.2]. Then $G$ is a minor of $L \oplus_{n} R$, which is itself a minor of $K_{m} \oplus_{n} K_{s}$, where $m=|L|$ and $s=|R|$. Let us abuse notation slightly, and define $K_{2} \oplus_{n} K_{s}:=K_{1} \oplus_{n+1} K_{s}$. By Lemma 3.4 and Proposition 3.6,

$$
l(G) \leqslant l\left(K_{m} \oplus_{n} K_{s}\right)= \begin{cases}1 & \text { if } m=s=2 \\ s-1 & \text { if } m=2<s \\ m+s-3 & \text { if } m, s>2 .\end{cases}
$$

Thus, in all cases, $l(G) \leqslant n-2$.

We remark that the proof of Proposition 3.14 (in conjunction with Proposition 3.14 itself) indicates that if $G$ is non-separable and odd bipartite, then $l(G)=n-2$.

A classification of graphs $G$ such that $l(G) \leqslant k$ for arbitrary $k$ seems beyond reach at the moment. However, since these graphs form a minor-closed class, we can determine whether $l(G) \leqslant k$ in time $O\left(n^{2}\right)[15]$. We show that in fact this can be done in linear time. Here, we consider a computational model where the atomic operations are integer operations. This is not only the consensus in the analysis of graph algorithms, but is also similar to the analysis of sorting algorithms, where typically the running time is given as the number of comparisons made (e.g. Merge-Sort makes $O(n \log n)$ comparisons; clearly each comparison could take $O(\log n)$ bitwise operations).

THEOREM 3.15. For any fixed $k$, deciding whether a connected graph $G$, given as an adjacency list, satisfies $l(G) \leqslant k$ can be done in $O(n)$ time.

Proof. Let us refer to a maximal path in $G$ consisting of vertices of degree 2 as a branch. If a branch does not belong to a non-separable block, then $G=L \oplus_{q} R$, where the branch is the path in the middle. We say that a branch is terminal if $L=K_{1}$ and non-terminal otherwise. We shall use the same notation as for Proposition 3.6.

The result is clear for $k=1$ (Proposition 3.11), so suppose $k \geqslant 2$. The algorithm goes as follows.

(1) If $n \leqslant(k+2)(k+1)(2 k-1)$, solve by brute force, i.e. by enumerating all elements of $\langle G\rangle$.

(2) If $G$ is a path, then return Yes.

(3) If $G$ has a vertex of degree at least $k+2$, then return No (Lemma 3.5).

(4) If $G$ has at least $4 k-1$ vertices of degree not 2, then return $N_{o}$ (Lemma 3.5(iii)).

(5) If $G$ has a non-separable block of size at least $k+3$, then return No (Proposition 3.14 and the remark after its proof).

(6) Let $P$ be the longest branch of $G$. If $P$ is terminal and has length at most $n-k-3$, or if $P$ is non-terminal and has length at most $n-k-4$, then return No. Otherwise, return Yes.

If the first five properties are not satisfied, then the number of vertices of degree 2 is at least

$$
n-t \geqslant(k+2)(k+1)(2 k-1)+1-(4 k-2)>(k+1)^{2}(2 k-1) .
$$

On the other hand, the number of branches is at most $(k+1) t / 2 \leqslant(k+1)(2 k-1)$. Thus the longest branch $P$ of $G$ has length $q \geqslant k+2$.

First, suppose that $P$ is terminal, i.e. $G=L \oplus_{q} R$ with $m=1$ and $s \geqslant 3$. If $q \leqslant n-k-3$, then $s-1=n-q-2 \geqslant k+1$ and $l(G) \geqslant l\left(G^{\prime}\right)=k+1$, where $G^{\prime}$ 
is the subgraph of $G$ induced by $L \cup P \cup\left\{r_{1}, \ldots, r_{k+2}\right\}$. Otherwise, $s \leqslant k+1$ hence $q \geqslant s$ and $l(G)=s-1 \leqslant k$.

Second, suppose that $P$ is non-terminal: i.e. $G=L \oplus_{q} R$ with $s \geqslant m \geqslant 3$. If $q \leqslant n-k-4$, then $m+s-3=n-q-3 \geqslant k+1$. Let

$$
\mu=\min \left\{m,\left\lfloor\frac{k+4}{2}\right\rfloor\right\} \quad \text { and } \quad \sigma=k+4-\mu \text {. }
$$

We then have

$$
\sigma+\mu-3=k+1, \quad q \geqslant k+2 \geqslant \sigma \geqslant \mu \geqslant 3, \quad m \geqslant \mu, \quad s \geqslant \sigma
$$

and $l(G) \geqslant l\left(G^{\prime}\right)=k+1$, where $G^{\prime}$ is the subgraph of $G$ induced by $\left\{l_{1}, \ldots, l_{\mu}\right\} \cup$ $P \cup\left\{r_{1}, \ldots, r_{\sigma}\right\}$. Otherwise, $m+s-3 \leqslant k$ hence $q \geqslant s$ and $l(G)=m+s-3 \leqslant k$.

Step 1 runs in $O(1)$ time; properties 2 to 4 are decidable in time $O(n)$. If the first four properties are not satisfied, the number $m$ of edges of $G$ is at most $\frac{1}{2}(k+1) t+$ $n-t \leqslant 2 n$. Then the following steps, which run in $O(n+m)$ (an algorithm to find the non-separable blocks in linear time is given in [10]), actually run in $O(n)$ time.

We now give an interpretation of Theorem 3.15 in terms of the pebble motion problem, reviewed in the introduction. In general, the problem of determining whether $S^{\prime}$ can be obtained from $S$ via a sequence of moves is NP-hard [19]. However, we can easily extend any pebble arrangement $T: P \rightarrow[n]$ into a singular transformation $\tau$ of $[n]$, by letting $\tau(p)=T(p)$ for all $p \in P$ and $\tau(q)=T(1)$ otherwise. Moreover, any sequence of moves corresponds to an element $\alpha \in\langle D\rangle$. Thus, $S^{\prime}$ can be obtained from $S$ via a sequence of moves if and only if the corresponding transformations satisfy $\sigma^{\prime}=\sigma \alpha$ for some $\alpha \in\langle D\rangle^{1}$ - in particular, if $D$ is strongly connected, then this is equivalent to $\sigma^{\prime} \mathcal{R} \sigma$. Theorem 3.15 then gives a linear-time algorithm to show that some arrangements cannot be obtained from $S$, solely based on how much they "shuffle" the pebbles. Indeed, for the sake of simplicity, let us assume that $\sigma$ and $\sigma^{\prime}$ have the same image, say $I$. The restriction of $\alpha$ to $I$ is then a permutation $\pi$, since $(p \sigma) \alpha=\left(p \sigma^{\prime}\right)$ for all $p \in P$. If $\pi$ has a cycle of length $k>l(D)$, then the algorithm in the proof of Theorem 3.15 will return No.

\section{Properties Related to Green's Relations}

In this section we characterise some semigroup theoretic properties of $\langle D\rangle$ in terms of certain digraph theoretic properties of $D$. In Proposition 3.1, we classified the digraphs $D$ for which $\langle D\rangle$ is $\mathcal{H}$-trivial. The purpose of this section is to give analogous classifications for Green's $\mathcal{R}$-, $\mathcal{L}$ - and $\mathcal{J}$-relations in Propositions 4.3, 4.4 and 4.5, respectively.

The proof of [14, Proposition 2.4.2] gives the following.

LEMma 4.1. Let $T$ be a subsemigroup of a semigroup $S$, let $a, b \in T$, and suppose $a, b$ are regular in $T$. Then the following hold:

(i) $a, b$ are $\mathcal{R}$-related in $T$ if and only if they are $\mathcal{R}$-related in $S$;

(ii) $a, b$ are $\mathcal{L}$-related in $T$ if and only if they are $\mathcal{L}$-related in $S$.

Recall that two elements of $\operatorname{Tran}_{n}$ are $\mathcal{R}-, \mathcal{L}$-, or $\mathcal{J}$ - related if and only if they have the same kernel, image, or rank, respectively.

Lemma 4.2. Let $D$ be a digraph. If $D$ contains a cycle and $(a \rightarrow b)$ is an arc in that cycle, then $(b \rightarrow a) \in\langle D\rangle$ and $(a \rightarrow b) \mathcal{R}(b \rightarrow a)$.

Proof. As noted earlier, it follows from [25, Lemma 2.3] that $(b \rightarrow a)$ belongs to $\langle D\rangle$. Since $(a \rightarrow b)$ and $(b \rightarrow a)$ are idempotents, and hence regular, and they have equal kernels, it follows follows from Lemma 4.1 that $(a \rightarrow b) \mathcal{R}(b \rightarrow a)$. 
Proposition 4.3. Let $D$ be a digraph. Then $\langle D\rangle$ is $\mathcal{R}$-trivial if and only if $D$ is acyclic.

Proof. $(\Rightarrow)$ It follows immediately from Lemma 4.2 that if $D$ contains a cycle, then it is not $\mathcal{R}$-trivial, and so the contrapositive of this implication holds.

$(\Leftarrow)$ Again we prove the contrapositive. Suppose that $\alpha, \beta \in\langle D\rangle$ are such that $\alpha \neq \beta$ and $\alpha \mathcal{R} \beta$. Then there exist $\gamma, \delta \in\langle D\rangle$ such that $\alpha \gamma=\beta$ and $\beta \delta=\alpha$, and there is $i \in[n]$ with $i \alpha \neq i \beta$. Hence $i \alpha \gamma=i \beta \neq i \alpha$ and $i \alpha=i \alpha \gamma \delta$. The former implies that $D$ contains a non-trivial path from $i \alpha$ to $i \alpha \gamma$, and the latter that $D$ contains a path from $i \alpha \gamma$ to $i \alpha$. Thus $D$ contains a cycle.

Proposition 4.4. Let $D$ be a digraph. Then $\langle D\rangle$ is $\mathcal{L}$-trivial if and only if the following hold:

(i) the out-degree of every vertex in $D$ is at most 1 ; and

(ii) $D$ contains no cycles of length greater than 2 .

Proof. $(\Rightarrow)$ We prove the contrapositive (i.e. that if either (i) or (ii) is not true, then $\langle D\rangle$ is not $\mathcal{L}$-trivial). If there are distinct $\operatorname{arcs} \alpha=(a \rightarrow b)$ and $\beta=(a \rightarrow c)$ in $D$, then $\alpha$ and $\beta$ are regular, and have the same image, and so $\alpha \mathcal{L} \beta$. If $D$ contains a cycle of length greater than 2 , then $\langle D\rangle$ is not $\mathcal{H}$-trivial, by Proposition 3.1, and hence it is not $\mathcal{L}$-trivial.

$(\Leftarrow)$ Suppose that both (i) and (ii) both hold. We begin by making some observations about the elements of $\langle D\rangle$ and their action on the vertices of $D$.

Suppose that $x_{0} \in[n]$ is an arbitrary vertex of $D$ with out-degree 1 . By the assumptions on the structure of $D$, there is a unique path

$$
x_{0} \rightarrow x_{1} \rightarrow \cdots \rightarrow x_{k-1} \rightarrow x_{k}
$$

in $D$ starting at $x_{0}$, and where $x_{k}$ has out-degree 0 or $\left(x_{k} \rightarrow x_{k-1}\right)$ is an arc in $D$. Since there are no vertices in $D$ with out-degree exceeding 1 , it follows that $\left(x_{i} \rightarrow x_{i+1}\right)$ is the only arc in $D$ starting at $x_{i}$ for every $i$. So, if $\gamma \in\langle D\rangle$, then

$$
x_{s} \gamma=x_{t} \text { and } s \leqslant t \text { for all } s \leqslant k-1 .
$$

First, we will show that

$$
\begin{aligned}
& \text { if } \gamma=\gamma_{0}\left(x_{0} \rightarrow x_{1}\right) \gamma_{1}\left(x_{1} \rightarrow x_{2}\right) \gamma_{2} \cdots \gamma_{r-1}\left(x_{r-1} \rightarrow x_{r}\right) \gamma_{r} \text { where } \gamma_{0}, \gamma_{1}, \ldots, \gamma_{r} \in\langle D\rangle \text {, } \\
& \text { then } x_{0} \gamma=x_{s} \text { where either } s \geqslant r \text { or } s=k-1 \text { and } r=k
\end{aligned}
$$

for all $0 \leqslant r \leqslant k$.

We proceed by induction on $r$. If $r=0$, then $\gamma=\gamma_{0}$ and $x_{0} \gamma=x_{l}$ for some $l \geqslant 0$ by (3). If $r>0$, then by induction there exists $l \geqslant r-1$ such that

$$
x_{0} \gamma_{0}\left(x_{0} \rightarrow x_{1}\right) \gamma_{1}\left(x_{1} \rightarrow x_{2}\right) \gamma_{2} \cdots \gamma_{r-1}=x_{l} .
$$

Suppose first that $l \geqslant r$. Then $x_{0} \gamma=x_{l}\left(x_{r-1} \rightarrow x_{r}\right) \gamma_{r}=x_{l} \gamma_{r}$. If $l \leqslant k-1$, then (3) gives $x_{l} \gamma_{r}=x_{s}$ for some $s \geqslant l \geqslant r$. If $l=k$, then by the form of $D, x_{l} \gamma_{r}=x_{k} \gamma_{r}$ can only be one of $x_{k}$ or $x_{k-1}$. On the other hand, if $l=r-1$, then $x_{0} \gamma=x_{r-1}\left(x_{r-1} \rightarrow\right.$ $\left.x_{r}\right) \gamma_{r}=x_{r} \gamma_{r}$, and $x_{r} \gamma_{r}=x_{m}$ where $m \geqslant r$, again by (3), unless $r=k$ in which case it is possible that $x_{r} \gamma_{r}=x_{k-1}$. This completes the proof of (4).

Second, suppose that $\gamma \in\langle D\rangle$ and $x_{0} \gamma=x_{r}$ for some $r$. Since there is a unique path from $x_{0}$ to $x_{r}$ in $D$, it follows that any factorisation of $\gamma$ in the arcs of $D$ must contain each of

$$
\left(x_{0} \rightarrow x_{1}\right),\left(x_{1} \rightarrow x_{2}\right), \ldots,\left(x_{r-1} \rightarrow x_{r}\right)
$$


in this order. In other words,

(5)

if $x_{0} \gamma=x_{r}$ for some $r$, then $\gamma=\gamma_{0}\left(x_{0} \rightarrow x_{1}\right) \gamma_{1}\left(x_{1} \rightarrow x_{2}\right) \gamma_{2} \cdots \gamma_{r-1}\left(x_{r-1} \rightarrow x_{r}\right) \gamma_{r}$

for some $\gamma_{0}, \gamma_{1}, \ldots, \gamma_{r} \in\langle D\rangle$.

We will now begin the proof of this implication in earnest. Suppose that there are $\alpha, \beta \in\langle D\rangle$ such that $\alpha \neq \beta$. We will show that $\alpha$ and $\beta$ are not $\mathcal{L}$-related. Since $\alpha \neq \beta$, there exists $x_{0} \in[n]$ such that $x_{0} \alpha \neq x_{0} \beta$. Since at least one of $\alpha, \beta$ does not fix $x_{0}$, it follows that the out-degree of $x_{0}$ is equal to 1 . Suppose that $x_{1}, \ldots, x_{k}$ are as in (2). From (3), $x_{0} \alpha=x_{r}$ and $x_{0} \beta=x_{s}$ for some $r, s \geqslant 0$. We may assume without loss of generality that $r>s$. We consider two cases separately.

CASE $1: s \leqslant k-2$ OR $\left(x_{k} \rightarrow x_{k-1}\right)$ IS NOT AN ARC IN D. By (5), $\alpha=\alpha_{0}\left(x_{0} \rightarrow x_{1}\right) \alpha_{1}\left(x_{1} \rightarrow x_{2}\right) \alpha_{2} \cdots \alpha_{r-1}\left(x_{r-1} \rightarrow x_{r}\right) \alpha_{r} \quad$ for some $\alpha_{0}, \alpha_{1}, \ldots, \alpha_{r} \in\langle D\rangle$. It follows that if $\gamma \in\langle D\rangle$ is such that $\beta=\gamma \alpha$, then

$$
\beta=\gamma \alpha=\left(\gamma \alpha_{0}\right)\left(x_{0} \rightarrow x_{1}\right) \alpha_{1}\left(x_{1} \rightarrow x_{2}\right) \alpha_{2} \cdots \alpha_{r-1}\left(x_{r-1} \rightarrow x_{r}\right) \alpha_{r} .
$$

But $x_{0} \beta=x_{s}$ and so (4) implies that $s \geqslant r$, contradicting the assumption that $s<r$. Hence $(\alpha, \beta) \notin \mathcal{L}$.

CASE 2: $s=k-1$ AND $\left(x_{k} \rightarrow x_{k-1}\right)$ IS AN ARC IN $D$. Since $r>s$, it follows that $x_{0} \alpha=x_{k}$ and $x_{0} \beta=x_{k-1}$. By (5), there exist $\alpha_{0}, \alpha_{1}, \ldots \alpha_{k} \in\langle D\rangle$ such that

$$
\alpha=\alpha_{0}\left(x_{0} \rightarrow x_{1}\right) \alpha_{1}\left(x_{1} \rightarrow x_{2}\right) \alpha_{2} \cdots \alpha_{k-1}\left(x_{k-1} \rightarrow x_{k}\right) \alpha_{k},
$$

and we may assume without loss of generality that $\alpha_{k}$ does not have $\left(x_{k} \rightarrow x_{k-1}\right)$ as a factor. Suppose that $\gamma \in\langle D\rangle$ is arbitrary. By (4),

$$
x_{0} \gamma \alpha_{0}\left(x_{0} \rightarrow x_{1}\right) \alpha_{1}\left(x_{1} \rightarrow x_{2}\right) \alpha_{2} \cdots \alpha_{k-2}\left(x_{k-2} \rightarrow x_{k-1}\right) \alpha_{k-1} \in\left\{x_{k-1}, x_{k}\right\} .
$$

In either case, $x_{0} \gamma \alpha=x_{k} \neq x_{k-1}=x_{0} \beta$ and so $\beta \neq \gamma \alpha$ for any $\gamma \in\langle D\rangle$, which implies $(\alpha, \beta) \notin \mathcal{L}$.

Proposition 4.5. Let $D$ be a digraph. Then the following are equivalent:

(i) $\langle D\rangle$ has at most one idempotent in every $\mathcal{L}$-class and every $\mathcal{R}$-class;

(ii) $\langle D\rangle$ is $\mathcal{J}$-trivial;

(iii) $D$ is acyclic and the out-degree of every vertex in $D$ is at most 1.

Proof. (i) $\Rightarrow$ (iii). If $D$ had a vertex of out-degree greater than 1 , then, as in the proof of Proposition $4.4,\langle D\rangle$ would contain two distinct $\mathcal{L}$-related idempotents.

If $D$ contained a cycle, then, by Lemma $4.2,\langle D\rangle$ would contain two distinct $\mathcal{R}$ related idempotents.

(iii) $\Rightarrow$ (ii). If $D$ is acyclic and the out-degree of every vertex in $D$ is at most 1 , then, by Propositions 4.3 and $4.4,\langle D\rangle$ is both $\mathcal{R}$ - and $\mathcal{L}$-trivial. Hence $\langle D\rangle$ is $\mathcal{J}$-trivial.

(ii) $\Rightarrow$ (i). Since $\langle D\rangle$ is $\mathcal{J}$-trivial, it is both $\mathcal{L}$ - and $\mathcal{R}$-trivial. Hence every $\mathcal{L}$ class and every $\mathcal{R}$-class contains exactly one element, and, in particular, at most one idempotent.

\section{OTHER CLASSICAL SEMIGROUP PROPERTIES}

A semigroup $S$ is called completely regular if every element belongs to subgroup. Equivalently, a semigroup is completely regular if and only if every element is $\mathcal{H}$ related to an idempotent. A finite semigroup $S$ is completely regular if and only if $x \mathcal{J} x^{2}$ for all $x \in S$.

If $D$ is any digraph with at most 2 vertices, then $\langle D\rangle$ is a band. Hence in the next two results we will assume that the number of vertices in $D$ is at least 3 . 
We say a digraph $D$ is directed-bipartite if there is a partition of the vertices $[n]$ of $D$ into two parts $V_{1}$ and $V_{2}$ such that every arc $(a \rightarrow b)$ of $D$ satisfies $a \in V_{1}$ and $b \in V_{2}$.

Proposition 5.1. Let $D$ be a connected digraph with at least 3 vertices. Then the following are equivalent:

(i) $\langle D\rangle$ is a band;

(ii) $\langle D\rangle$ is completely regular;

(iii) $D$ is directed-bipartite.

Proof. (i) and (iii) are shown to be equivalent in [5, Theorem 2.12].

(i) $\Rightarrow$ (ii). This implication follows immediately, since every band is completely regular.

(ii) $\Rightarrow$ (iii). We prove the contrapositive. Suppose that $D$ contains the arcs $(a \rightarrow b)$ and $(b \rightarrow c)$, where $a, b, c \in[n]$ are distinct, and consider $\alpha=(b \rightarrow c)(a \rightarrow b)$. Then $\operatorname{rk}(\alpha)=n-1$ and $\operatorname{rk}\left(\alpha^{2}\right)=n-2$, and so $\alpha$ and $\alpha^{2}$ are not $\mathcal{J}$-related in $\operatorname{Tran}_{n}$. It follows that $\alpha$ and $\alpha^{2}$ are not $\mathcal{J}$-related in $\langle D\rangle$, and so $\langle D\rangle$ is not completely regular.

COROLlary 5.2. Let D be a connected acyclic digraph with at least 3 vertices. Then the following are equivalent:

(i) $\langle D\rangle$ is a band;

(ii) $\langle D\rangle$ is completely regular;

(iii) $\langle D\rangle$ is regular;

(iv) $D$ is directed-bipartite.

Proof. It suffices to prove that (iii) implies (i), so we suppose that $\langle D\rangle$ is regular. Since $D$ is acyclic, $\langle D\rangle$ is $\mathcal{R}$-trivial by Lemma 4 .3. Since $\langle D\rangle$ is regular, it follows that every $\mathcal{R}$-class contains an idempotent. But every $\mathcal{R}$-class is of size 1 , and so every element of $\langle D\rangle$ is an idempotent. In other words, $\langle D\rangle$ is a band.

There are non-acyclic digraphs $D$ such that $\langle D\rangle$ is regular but not a band. For example, if $D$ is any strong tournament with $n \geqslant 3$ vertices, then $\langle D\rangle=\operatorname{Sing}_{n}$, and Sing $_{n}$ is regular but not a band.

For $n \geqslant 1$, an $n$-fan is a connected acyclic digraph isomorphic to the digraph with $\operatorname{arcs}(i, n)$ for all $i \in\{1, \ldots, n-1\}$. A 1 -fan is just a one-vertex digraph with no arcs. A picture of an $n$-fan can be seen in Figure 5 .

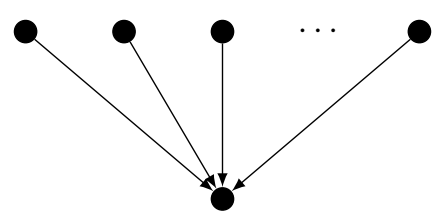

FiguRE 5. An $n$-fan.

A semigroup $S$ is called inverse if for all $x \in S$ there exists a unique $y \in S$ such that $x y x=x$ and $y x y=y$. It is well-known (see, for example, [14, Theorem 5.1.1]) that a semigroup $S$ is inverse if and only if it is regular and its idempotents commute. The same theorem from [14] also says that $S$ is inverse if and only if each $\mathcal{R}$-class and each $\mathcal{L}$-class of $S$ contains exactly one idempotent. A semilattice is a semigroup of commuting idempotents. For any set $X$, the power set $2^{X}=\{A: A \subseteq X\}$ of $X$ is a semilattice under $\cup$; the subsemigroup $2^{X} \backslash\{\varnothing\}$ is called the free semilattice of degree $|X|$. If $X_{1}, \ldots, X_{k}$ are disjoint finite sets, then $\left(2^{X_{1}} \times \cdots \times 2^{X_{k}}\right) \backslash\{(\varnothing, \ldots, \varnothing)\}$ is a free semilattice of degree $\left|X_{1}\right|+\cdots+\left|X_{k}\right|$, isomorphic to $2^{X_{1} \cup \cdots \cup X_{k}} \backslash\{\varnothing\}$. 
Proposition 5.3. Let $D$ be a connected digraph on $n$ vertices. Then the following are equivalent:

(i) $\langle D\rangle$ is a free semilattice of degree $n-1$;

(ii) $\langle D\rangle$ is inverse;

(iii) $\langle D\rangle$ is commutative;

(iv) $D$ is a fan.

If any of the above conditions holds, then $|\langle D\rangle|=2^{n-1}-1$.

Proof. (i) $\Rightarrow$ (ii). Every semilattice is an inverse semigroup.

(ii) $\Rightarrow$ (iii). Since $\langle D\rangle$ is inverse, it has exactly one idempotent in every $\mathcal{L}$ - and $\mathcal{R}$-class, and hence by Proposition $4.5,\langle D\rangle$ is $\mathcal{J}$-trivial. Thus $\langle D\rangle$ is a semilattice, and hence it is commutative.

(iii) $\Rightarrow$ (iv). Assume that $\langle D\rangle$ is commutative. If $D$ contains distinct arcs $\alpha=$ $(a \rightarrow b)$ and $\beta=(b \rightarrow c)$, then $\alpha \beta \neq \beta \alpha$, a contradiction. If $D$ contains distinct arcs $\gamma=(d \rightarrow e)$ and $\delta=(d \rightarrow f)$, then $\gamma \delta \neq \delta \gamma$, a contradiction. Since $D$ is connected, it follows that $D$ is a fan.

(iv) $\Rightarrow$ (i). We may assume that the unique sink in $D$ is the vertex $n$. If $S$ is any subset of $\{1, \ldots, n-1\}$, then we define $\alpha_{S} \in \operatorname{Tran}_{n}$ by

$$
v \alpha_{S}= \begin{cases}n & \text { if } v \in S, \\ v & \text { if } v \notin S .\end{cases}
$$

If $S=\left\{s_{1}, \ldots, s_{k}\right\}$ is not empty, then $\alpha_{S}=\left(s_{1} \rightarrow n\right) \cdots\left(s_{k} \rightarrow n\right) \in\langle D\rangle$. Conversely, the arcs of $D$ commute and so any transformation in $\langle D\rangle$ is of the form $\alpha_{S}$ for some non-empty subset $S$ of $\{1, \ldots, n-1\}$. If $S$ and $T$ are non-empty subsets of $\{1, \ldots, n-1\}$, then it is routine to verify that $\alpha_{S} \alpha_{T}=\alpha_{S \cup T}$. It follows that the map $\phi: 2^{\{1, \ldots, n-1\}} \backslash\{\varnothing\} \rightarrow\langle D\rangle$ defined by $S \phi=\alpha_{S}$ is an isomorphism.

If $D$ is a digraph with connected components $D_{1}, \ldots, D_{r}$, then it follows from Proposition 2.1 that $\langle D\rangle$ is inverse if and only if each $\left\langle D_{i}\right\rangle$ is inverse. From this we obtain the following corollary to Proposition 5.3.

COROLlary 5.4. The number of digraphs (up to isomorphism) with $n$ vertices such that $\langle D\rangle$ is an inverse semigroup is equal to $n-1$.

Proof. Suppose that $\langle D\rangle$ is inverse, that the connected components of $D$ are $D_{1}, \ldots, D_{r}$, and write $d_{i}=\left|D_{i}\right|$ for each $i$. It follows from Proposition 5.3 that each $D_{i}$ is a fan, and each $\left\langle D_{i}\right\rangle$ is a free semilattice of degree $d_{i}-1$. It follows that $\langle D\rangle$ is a free semilattice of degree $\left(d_{1}-1\right)+\cdots+\left(d_{r}-1\right)=n-r$. So the isomorphism class of $\langle D\rangle$ is completely determined by $r$, the number of connected (fan) components of $D$. Since $r$ can take any value from 1 to $n-1$, the proof is complete.

\section{ZEROS}

An element $a$ of a semigroup $S$ is a left zero if $a b=a$ for all $b \in S$. Right zeros are defined analogously. An element is a zero if it is both a left and right zero. If a semigroup has a left zero and a right zero, then it has a unique zero. In this section, we obtain necessary and sufficient conditions on a digraph $D$ so that $\langle D\rangle$ has various properties associated to left or right zeros. Some of our results also classify the digraphs $D$ for which $\langle D\rangle$ consists of a single $\mathcal{R}$-, $\mathcal{L}$-, or $\mathcal{J}$-class (see Proposition 2.2 for the analogous result for the $\mathcal{H}$-relation). We begin with two technical lemmas.

LEMMA 6.1. If $D$ is strongly connected, then $\langle D\rangle$ contains every constant map. 
Proof. Since $D$ is strongly connected, it suffices to show that $\langle D\rangle$ contains any constant map. Suppose that there exists $\alpha \in\langle D\rangle$ with rank $r$, where $2 \leqslant r \leqslant n-1$. We prove that there exists $\beta \in\langle D\rangle$ with $\operatorname{rk}(\beta)<\operatorname{rk}(\alpha)$, from which the lemma follows. Since $\operatorname{rk}(\alpha) \geqslant 2$, there exist distinct $i, j \in \operatorname{im}(\alpha)$, and since $D$ is strongly connected, there is a path in $D$ from $i$ to $j$. If $(i \rightsquigarrow j)$ denotes the product of the arcs in a path from $i$ to $j$, then it is routine to check that $\operatorname{rk}(\alpha(i \rightsquigarrow j))<\operatorname{rk}(\alpha)$, as required.

Lemma 6.2. If $D$ is connected, then there exists $\alpha \in\langle D\rangle$ such that $i \alpha$ belongs to a terminal component of $D$ for each $i \in[n]$.

Proof. Suppose without loss of generality that $\{1, \ldots, k\}$ are the vertices of $D$ that do not belong to a terminal component of $D$. Then for every $i \in\{1, \ldots, k\}$ there exists a vertex $t_{i}$ in a terminal component of $D$ such that there is a path in $D$ from $i$ to $t_{i}$. The product $\alpha=\prod_{i=1}^{k}\left(i \rightsquigarrow t_{i}\right)$ has the required property.

Proposition 6.3. Let $D$ be a connected digraph. Then the following hold:

(i) $\langle D\rangle$ has a left zero if and only if all terminal components of $D$ are trivial. If this is the case, then $\alpha$ is a left zero of $\langle D\rangle$ if and only if va belongs to a terminal component for all vertices $v$.

(ii) $\langle D\rangle$ has a right zero if and only if it has exactly one terminal component. If this is the case, then $\alpha$ is a right zero of $\langle D\rangle$ if and only if it is a constant map.

(iii) $\langle D\rangle$ has a zero if and only if it has exactly one terminal component $T$, which is trivial. If this is the case, then the zero of $\langle D\rangle$ is the constant map with image $T$.

Proof. (i). Suppose that $\alpha \in\langle D\rangle$ is a left zero, let $T$ be an arbitrary terminal component, and let $a, b \in T$. By Lemma 6.1 , there exist $\beta, \gamma \in\langle D\rangle$ such that $t \beta=a$ and $t \gamma=b$ for all $t \in T$. Let $t \in T$ be arbitrary. Since $T$ is terminal, $t \alpha \in T$. But then, since $\alpha$ is a left zero, $a=(t \alpha) \beta=t(\alpha \beta)=t \alpha=t(\alpha \gamma)=(t \alpha) \gamma=b$. This shows that $|T|=1$.

Conversely, suppose that all terminal components of $D$ are trivial: $\left\{t_{1}\right\}, \ldots,\left\{t_{k}\right\}$. By Lemma 6.2, there exists $\alpha \in\langle D\rangle$ such that $v \alpha$ belongs to a terminal component for each vertex $v \in[n]$. Now let $\beta \in\langle D\rangle$ be arbitrary. Let $v \in[n]$ and put $v \alpha=t_{j}$. Then $v \alpha \beta=t_{j} \beta=t_{j}=v \alpha$, so that $\alpha \beta=\alpha$, and $\alpha$ is a left zero.

On the other hand, suppose that all of the terminal components of $D$ are trivial, and that $\alpha \in\langle D\rangle$ is such that $v \alpha$ does not belong to a terminal component for some $v \in[n]$. Then $D$ contains some $\operatorname{arc}(v \alpha \rightarrow j)$. But then $\alpha \neq \alpha(v \alpha \rightarrow j)$, so that $\alpha$ is not a left zero.

(ii). Suppose that $D$ has at least two terminal components. Then there exist distinct terminal components $T_{1}$ and $T_{2}$ and a vertex $v$ such that there is a path from $v$ to a vertex from $T_{1}$ and a path from $v$ to a vertex from $T_{2}$. For $i=1,2$, let $\beta_{i} \in\langle D\rangle$ be such that $v \beta_{i} \in T_{i}$. If $\alpha \in\langle D\rangle$ is a right zero, then

$$
v \alpha=v \beta_{1} \alpha \in T_{1} \quad \text { and } \quad v \alpha=v \beta_{2} \alpha \in T_{2},
$$

which is the desired contradiction.

Conversely, suppose that $D$ has only one terminal component $T$ and fix some $t \in T$. By Lemmas 6.1 and 6.2, there exist $\alpha_{1}, \alpha_{2} \in\langle D\rangle$ such that $\operatorname{im}\left(\alpha_{1}\right) \subseteq T$, and $T \alpha_{2}=$ $\{t\}$. Then $\alpha_{1} \alpha_{2} \in\langle D\rangle$ is a constant map (with image $\{t\}$ ), and hence a right zero.

On the other hand, suppose that $D$ only has one terminal component $T$, and that $\alpha \in\langle D\rangle$ is not a constant map. So $\operatorname{rk}(\alpha) \geqslant 2$. We know that $\langle D\rangle$ contains some constant map $\beta$. But then $\operatorname{rk}(\beta \alpha)=\operatorname{rk}(\beta)=1 \neq \operatorname{rk}(\alpha)$, so that $\beta \alpha \neq \alpha$, whence $\alpha$ is not a right zero. 
A semigroup $S$ is called a left zero semigroup if every element of $S$ is a left zero; right zero semigroups are defined analogously.

Proposition 6.4. Let $D$ be a digraph. Then the following are equivalent:

(i) $\langle D\rangle$ is a left zero semigroup;

(ii) $\langle D\rangle$ has a unique $\mathcal{L}$-class;

(iii) there is a unique non-trivial connected component $K$ of $D$, and

$$
K^{-1}=\{(y \rightarrow x):(x \rightarrow y) \in K\}
$$

is a fan.

Proof. (i) $\Rightarrow$ (ii). Every left zero semigroup has a unique $\mathcal{L}$-class.

(iii) $\Rightarrow$ (iii). Since $\langle D\rangle$ has a unique $\mathcal{L}$-class, it follows that all of the arcs in $D$ belong to the same $\mathcal{L}$-class. Hence the $\operatorname{arcs}$ in $D$ have the same image: say, $[n] \backslash\{x\}$ for some fixed $x \in[n]$. In other words, the $\operatorname{arcs}$ in $D$ are all of the form $(x \rightarrow y)$, $y \neq x$.

(iii) $\Rightarrow$ (i). If the only arcs in $D$ are of the form $(x \rightarrow y)$ for some fixed $x$, then $(x \rightarrow y)(x \rightarrow z)=(x \rightarrow y)$ for all $y, z$, and so $\langle D\rangle=D$ is a left zero semigroup.

Proposition 6.5. Let $D$ be a digraph. Then the following are equivalent:

(i) $\langle D\rangle$ is a right zero semigroup;

(ii) $\langle D\rangle$ has a unique $\mathcal{R}$-class;

(iii) there is a unique non-trivial connected component $K$ of $D$, and $K$ has 2 vertices.

Proof. (i) $\Rightarrow$ (ii). Every right zero semigroup has a unique $\mathcal{R}$-class.

(ii) $\Rightarrow$ (iii). Since $\langle D\rangle$ has a unique $\mathcal{R}$-class, all elements of $D$ are $\mathcal{R}$-related. But $(i \rightarrow j) \mathcal{R}(k \rightarrow l)$ if and only if $\{i, j\}=\{k, l\}$ and so all of the arcs of $D$ involve the same two vertices.

(iii) $\Rightarrow$ (i). In this case, $\langle D\rangle$ is isomorphic to a subsemigroup of Sing $_{2}$, which is a right zero semigroup, and hence $\langle D\rangle$ is a right zero semigroup also.

Recall that a semigroup is simple if it contains a single $\mathcal{J}$-class.

Proposition 6.6. Let $D$ be a connected digraph. Then the following are equivalent:

(i) $\langle D\rangle$ is a rectangular band;

(ii) $\langle D\rangle$ is simple;

(iii) $\langle D\rangle$ is a left or right zero semigroup.

Proof. (iii) $\Rightarrow$ (ii). Every left or right zero semigroup is simple.

(ii) $\Rightarrow$ (i). If $\langle D\rangle$ is simple, then it is completely regular. If $n \geqslant 3$, then we conclude from Proposition 5.1 that $\langle D\rangle$ is a band; this is also true if $n \leqslant 2$. Every simple band is a rectangular band.

(i) $\Rightarrow$ (iii). Suppose that $\langle D\rangle$ is a rectangular band. Since $\langle D\rangle$ has a single $\mathcal{J}$-class, every element of $\langle D\rangle$ has rank $n-1$. It follows that $\langle D\rangle$ consists entirely of arcs. If $\langle D\rangle$ is not a left or right zero semigroup, then there exist distinct $\operatorname{arcs} \alpha, \beta, \gamma \in\langle D\rangle$ such that $\alpha \mathcal{R} \beta$ and $\beta \mathcal{L} \gamma$. The former implies that $\alpha=(a \rightarrow b)$ and $\beta=(b \rightarrow a)$ for some $a, b$, and the latter that $\gamma=(b \rightarrow c)$ for some $c$, where $a, b, c \in[n]$ are distinct. But then $\gamma \alpha \in\langle D\rangle$ is not an idempotent, a contradiction.

Recall that a semigroup $S$ with a zero element 0 is 0 -simple if $S^{2} \neq\{0\}$ and its $\mathcal{J}$-classes are $\{0\}$ and $S \backslash\{0\}$. 
Proposition 6.7. Let $D$ be a digraph. Then $\langle D\rangle$ is 0-simple if and only if the only non-trivial connected component of $D$ is one of the following:
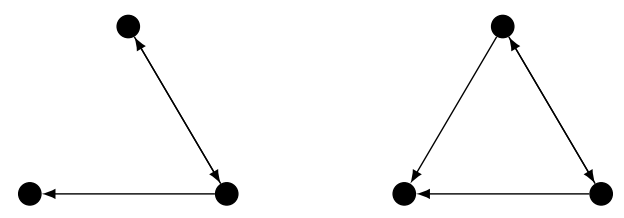

Proof. $(\Leftarrow)$ It is straightforward to verify that $\langle D\rangle$ is 0 -simple if $D$ is either of the given digraphs, using GAP [22] for instance.

$(\Rightarrow)$ Suppose that $\langle D\rangle$ is 0 -simple and, without loss of generality, that $D$ has no isolated vertices. Since $\langle D\rangle$ is 0 -simple, it has two $\mathcal{J}$-classes, and the minimum one contains only the zero element. In particular, $\langle D\rangle$ contains at most one element of rank smaller than $n-1$ and no elements of rank smaller than $n-2$.

Suppose that $D$ has two connected components $D_{1}$ and $D_{2}$. If there is an arc $\alpha$ in $D_{1}$, and distinct arcs $\beta, \gamma$ in $D_{2}$, then $\alpha \beta, \alpha \gamma \in\langle D\rangle$ are distinct elements of rank $n-2$, a contradiction. Hence, if $D$ has more than one connected component, then every connected component has exactly one arc. In this case, $\langle D\rangle$ contains at least 2 arcs and the zero element and so $|\langle D\rangle| \geqslant 3$. But Proposition 4.5 implies that $\langle D\rangle$ is $\mathcal{J}$-trivial and so $\langle D\rangle$ has at least three $\mathcal{J}$-classes, a contradiction. Thus $D$ is connected.

If $\alpha \in\langle D\rangle$ is the zero element, then by Proposition 6.3, $\alpha$ is constant which implies that $1=\operatorname{rk}(\alpha) \geqslant n-2$, and so $n \leqslant 3$. It is possible to check that if $D^{\prime}$ is any digraph with at most 3 vertices such that $\left\langle D^{\prime}\right\rangle$ is 0 -simple, then $D^{\prime}$ is isomorphic to one of the two given digraphs.

Proposition 6.8. Let $D$ be a digraph. Then $\langle D\rangle$ is congruence-free if and only if the only non-trivial connected component of $D$ is one of the following:
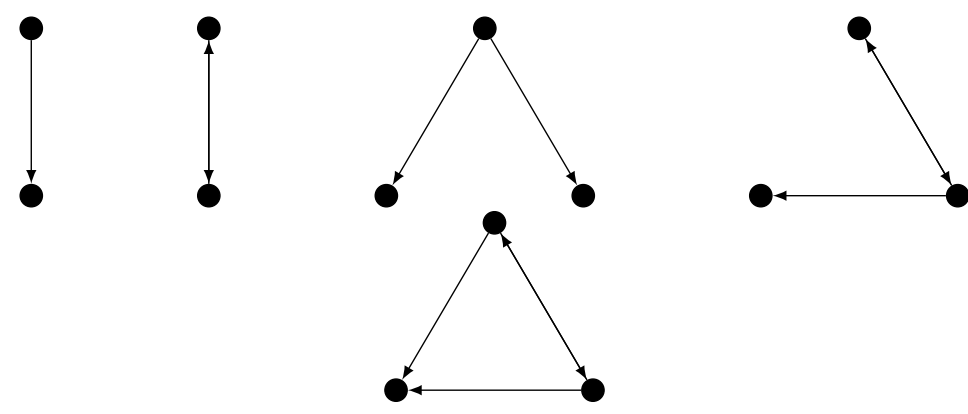

Proof. Let $D_{1}, D_{2}, D_{3}, D_{4}, D_{5}$ (left to right) be the digraphs in the statement of the proposition.

$(\Leftarrow)$ The semigroups $\left\langle D_{1}\right\rangle,\left\langle D_{2}\right\rangle,\left\langle D_{3}\right\rangle$ have size at most 2 , and so are congruencefree. It is straightforward to verify that $\left\langle D_{4}\right\rangle$ and $\left\langle D_{5}\right\rangle$ are both congruence-free (using GAP [22] for instance).

$(\Rightarrow)$ If $\langle D\rangle$ is congruence-free, then either $|\langle D\rangle| \leqslant 2,\langle D\rangle$ is 0-simple, or $\langle D\rangle$ is a simple group; see [14, Theorems 3.7.1 and 3.7.2]. So, by Propositions 2.2 and 6.7, it suffices to note that the only digraphs $D$ so that $|\langle D\rangle|=2$ are the digraphs $D_{2}$ and $D_{3}$.

\section{REFERENCES}

[1] A. Ya. Aŭzenshtat, The defining relations of the endomorphism semigroup of a finite linearly ordered set, Sib. Mat. Zh. 3 (1962), 161-169. 
[2] Jørgen Bang-Jensen and Gregory Z. Gutin, Digraphs, Springer Monographs in Mathematics, Springer London, 2009.

[3] A. V. Bankevich and Dmitriy V. Karpov, Bounds of the number of leaves of spanning trees, Journal of Mathematical Sciences 184 (2012), no. 5, 564-572.

[4] Adrian Bondy, Graph Theory, Graduate Texts in Mathematics, Springer, 2008.

[5] P. J. Cameron, A. Castillo-Ramirez, M. Gadouleau, and J. D. Mitchell, Lengths of words in transformation semigroups generated by digraphs, Journal of Algebraic Combinatorics 45 (2017), no. 1, 149-170.

[6] Igor Dolinka and James East, Idempotent generation in the endomorphism monoid of a uniform partition, Communications in Algebra 44 (2016), no. 12, 5179-5198.

[7] Olexandr Ganyushkin and Volodymyr Mazorchuk, Classical finite transformation semigroups, Algebra and Applications, vol. 9, Springer London, 2009.

[8] Gracinda M. S. Gomes and John M. Howie, On the ranks of certain finite semigroups of transformations, Mathematical Proceedings of the Cambridge Philosophical Society 101 (1987), no. 3, 395-403.

[9] Peter M. Higgins, Combinatorial results for semigroups of order-preserving mappings, Mathematical Proceedings of the Cambridge Philosophical Society 113 (1993), no. 2, 281-296.

[10] John Hopcroft and Robert Tarjan, Algorithm 447: efficient algorithms for graph manipulation, Communications of the ACM 16 (1973), no. 6, 372-378.

[11] Gábor Horváth, Chrystopher L. Nehaniv, and Károly Podoski, The maximal subgroups and the complexity of the flow semigroup of finite (di)graphs, International Journal of Algebra and Computation 27 (2017), no. 7, 863-886.

[12] John M. Howie, The subsemigroup generated by the idempotents of a full transformation semigroup, J. London Math. Soc. 41 (1966), no. 1, 707-716.

[13] _ Idempotent generators in finite full transformation semigroups, Proceedings of the Royal Society of Edinburgh: Section A Mathematics 81 (1978), no. 3-4, 317-323.

[14] _ Fundamentals of semigroup theory, London Mathematical Society Monographs. New Series, vol. 12, The Clarendon Press Oxford University Press, New York, 1995, Oxford Science Publications.

[15] Ken-ichi Kawarabayashi, Yusuke Kobayashi, and Bruce Reed, The disjoint paths problem in quadratic time, Journal of Combinatorial Theory, Series B 102 (2012), no. 2, 424-435.

[16] Daniel Kornhauser, Gary Miller, and Paul Spirakis, Coordinating pebble motion on graphs, the diameter of permutation groups, and applications, 25th Annual Symposium on Foundations of Computer Science, 1984., IEEE, 1984, pp. 241-250.

[17] James D. Mitchell et al., Semigroups - GAP package, Version 3.1.1, 2019.

[18] Jean Eric Pin, Varieties of Formal Languages, Foundations of Computer Science, Springer, 1986.

[19] Daniel Ratner and Manfred Warmuth, The $\left(n^{2}-1\right)$-puzzle and related relocation problems, Journal of Symbolic Computation 10 (1990), no. 2, 111-137.

[20] John Rhodes, Applications of automata theory and algebra: Via the mathematical theory of complexity to biology, physics, psychology, philosophy, and games, World Scientific Pub Co Inc, 2009.

[21] Andrew Solomon, Catalan monoids, monoids of local endomorphisms, and their presentations, Semigroup Forum 53 (1996), no. 1, 351-368.

[22] The GAP Group, GAP - Groups, Algorithms, and Programming, Version 4.10.1, 2019, https: //www.gap-system.org.

[23] Richard M. Wilson, Graph puzzles, homotopy, and the alternating group, Journal of Combinatorial Theory, Series B 16 (1974), no. 1, 86-96.

[24] E. M. Wright, The number of irreducible tournaments, Glasgow Mathematical Journal 11 (1970), no. 2, 97-101.

[25] Xiuliang Yang and Haobo Yang, Maximal regular subsemibands of $\operatorname{Sing}_{n}$, Semigroup Forum 72 (2005), no. 1, 75-93.

[26] _ Isomorphisms of transformation semigroups associated with simple digraphs, AsianEuropean Journal of Mathematics 2 (2009), no. 4, 727-737. 
James EAst, Centre for Research in Mathematics, School of Computing, Engineering and Mathematics, Western Sydney University, Locked Bag 1797, Penrith NSW 2751, Australia. E-mail : j.east@westernsydney.edu.au

Maximilien Gadouleau, Department of Computer Science, Durham University, South Road, Durham, DH1 3LE, UK.

E-mail : m.r.gadouleau@durham.ac.uk

James D. Mitchell, School of Mathematics and Statistics, University of St Andrews, St Andrews, Fife KY16 9SS, UK.

E-mail : jdm3@st-andrews.ac.uk 